Mon. Not. R. Astron. Soc. 000, 000-000 (0000) Printed 28 September $2018 \quad$ (MN LATEX style file v2.2)

\title{
Variability of QSOs with variable regions in broad absorption troughs from the Sloan Digital Sky Survey
}

\author{
Zhi-Cheng He, Wei-Hao Bian ${ }^{\star}$, Xiao-Lei Jiang and Xue Ge \\ Department of Physics and Institute of Theoretical Physics, Nanjing Normal University, Nanjing 210023, China
}

28 September 2018

\begin{abstract}
The variability of broad absorption lines is investigated for a sample of 188 broad-absorptionline (BAL) quasars (QSOs) $(z>1.7)$ with at least two-epoch observations from the Sloan Digital Sky Survey Data Release 7 (SDSS DR7), covering a time-scale of about $0.001-3$ years in the rest frame. Considering only the longest time-scale between epochs for each QSO, 73 variable regions in the C IV BAL troughs are detected for 43 BAL QSOs. The proportion of BAL QSOs showing variable regions increases with longer time-interval than about 1 year in the rest frame. The velocity width of variable regions is narrow compared to the BALtrough outflow velocity. For 43 BAL QSOs with variable regions, it is found that there is a medium strong correlation between the variation of the continuum luminosity at $1500 \AA$ and the variation of the spectral index. With respect to the total 188 QSOs, larger proportion of BAL QSOs with variable regions appears bluer during their brighter phases, which implies that the origin of BAL variable regions is related to the central accretion process. For $43 \mathrm{BAL}$ QSOs with variable regions, it is possible that there is a negative medium strong correlation between the absolute variation of the equivalent width and the Mg II -based black hole mass, and a medium strong correlation between the maximum outflow velocity of variable regions and the Eddington ratio. These results imply the connection between the BAL-trough variation and the central accretion process.
\end{abstract}

Key words: galaxies:active—galaxies:nuclei—quasars:absorption lines

\section{INTRODUCTION}

Broad absorption line quasars (BAL QSOs) exhibit broad absorption troughs for high-ionization ultraviolet (UV) lines such as $\mathrm{Si}$ ' IV 1399, C IV 1549, C III] 1909 (known as HiBAL), or/and lowionization UV lines such as Mg II 2799 (known as LoBAL). BAL QSOs were usually identified from their UV spectra by the balnicity index (BI), considering different BAL outflow velocity and/or BAL velocity width (e.g. Weymann et al. 1991; Trump et al. 2006; Gibson et al. 2009; He et al. 2014). BAL troughs are present in about 10-40\% of QSOs (e.g. Gibson et al. 2009; Allen et al. 2011).

BAL troughs in QSOs are thought to be the strongest observed signature of QSO winds (Fabian 2012). One explanation for BALs is an orientation-dependent effect, where QSOs appear as BAL QSOs when the disk wind is on the line of sight (e.g. Murray et al. 1995; Elvis 2002). The requirement that detection of a wind should be orientation-dependent is very similar to the case of other structures in QSOs, such as the broad-line region (BLR) or jet (e.g. Urry \& Padovani 1995). Another explanation for BALs is an orientation-independent evolution effect (e.g. Gibson et al. 2008; Zubovas \& King 2013). As an evolutionary stage of active galactic

* E-mail:whbian@njnu.edu.cn nucleus (AGN), the expulsion of gas and dust by galaxy collision possibly causes the BAL outflows. It was supported by no correlations exist between outflow properties and orientation, such as the similar range of viewing angles for radio-loud BALs and radio-loud non-BALs (e.g. Fine et al. 2011; Bruni et al. 2012).

The disk wind in BAL QSOs is believed to come from the central supermassive black hole (SMBH) accretion disc, and BAL region often lies outside the BLR region (e.g. Proga et al. 2000; Murray et al. 1995; Filiz Ak et al. 2013). There exists an empirical relation between the BLR size and the continuum luminosity in AGN/QSOs (e.g. Kaspi et al. 2005; Bentz et al. 2009). For the BAL region outside of BLR region, larger luminosity would lead to larger size, smaller orbital velocity of the BAL regions. It would lead the properties/variability of BAL outflows as a function of luminosity. The wind dependence on the properties of QSOs has been discussed by many authors, suggesting the connection between the outflow and the accretion process (e.g. Laor \& Brandt 2002; Ganguly et al. 2007; Baskin \& Laor 2013; He et al. 2014). It was found that the maximum outflow velocity increases with both the bolometric luminosity and the blueness of the spectral slope, suggesting the idea of radiation-pressuredriven outflows (Laor \& Brandt 2002; Ganguly et al. 2007). At the same time, BAL-troughs often vary over rest-frame time- 
scales of days to years, which can provide clues to the origin of BAL QSOs (e.g. Gibson et al.|2008; Capellupo et al. 2011, 2012, 2013; Filiz Ak et al.2012,2013; Grier et al.2015). Capellupo et al. (2011, 2012, 2013) investigated BAL variability for 24 QSOs with long time-scales and multiple epochs. They found that variability typically occurs only in portions of the BAL troughs; the components at higher outflow velocities are more likely to vary than those at lower velocities and weaker BALs are more likely to vary than stronger BALs; both the incidence and the amplitude of variability are greater across longer time-scales. With a sample of 291 BAL QSOs from SDSS-I/II/III, Filiz Ak et al. (2013) investigated BAL variations as a function of QSOs properties and did not find significant evidence for correlations between BAL variability and luminosity/Eddington ratio/SMBH mass. For C IV 1549 troughs on moderate time-scales (1-2.5 yrs), they suggested possible correlations between BAL variability and luminosity/Eddington ratio. Filiz Ak et al. (2013) used C IV emission line to calculate the SMBH mass and the Eddington ratio, which have larger scatters and bias compared to that by other emission lines such as $\mathrm{H} \beta, \mathrm{Mg}$ II .

For some samples of QSOs, it has been shown that spectra of QSOs at low redshifts are bluer during their brighter phases (e.g. Wilhite et al. 2005; Pu et al.2006; Meusinger et al.2011; Zuo et al. 2012; Bian et al. 2012a). The trend of bluer spectra during brighter phases is usually explained by the accretion variation. During the brighter phase, the accretion disk becomes hotter and its emission peak would move to shorter wavelengths (big blue bump), which would also lead to larger variance in the blue spectrum. However, other contributions may mingle in this kind of investigation, such as the contribution of UV/optical Fe II , Balmer continuum, jet, and the host, as well as the complex of accretion disk model. With two-epoch variation, it was found that the spectra of half of the QSOs appear redder during their brighter phases (Bian et al.2012a; Guo \& Gu 2014).

The feature of variable regions in BAL-trough were investigated by some authors (e.g. Gibson et al.2008; Filiz Ak et al.2012, 2013). Using a sample of 13 BAL QSOs with two-epoch spectra covering 3-6 years in the rest frame, Gibson et al. (2008) identified the variable regions in BAL-trough and investigated the variation of C IV BAL-trough between the Large Bright Quasar Survey (LBQS; Hewett et al. 1995) and the Sloan Digital Sky Survey (SDSS). With SDSS/Baryon Oscillation Spectroscopic Survey (BOSS), we have investigated the relation between the wind and QSO properties for a single BAL QSO with 18 epochs observations covering about 3 years in the rest frame (He et al. 2014). Here, we present a sample of 188 BAL QSOs with at least two-epoch observation from SDSS Data Release 7 (DR7). This sample is used to investigate the feature of the BAL-trough variable regions, the variability of UV spectral index and the BAL-trough equivalent width (EW), as well as the relation with the central accretion properties. Section 2 presents the sample. Section 3 gives the spectral analysis. Section 4 contains our results and discussion. A summery is given in the last section. Throughout this work, we use a cosmology with $H_{0}=70 \mathrm{~km} \mathrm{~s}^{-1} \mathrm{Mpc}^{-1}, \Omega_{M}=0.3$, and $\Omega_{\Lambda}=0.7$.

\section{THE SAMPLE OF C IV BAL QSOS WITH TWO-EPOCH OBSERVATIONS FROM THE SDSS DR7}

The SDSS DR7 (York et al. 2000) contains imaging of almost $11663 \mathrm{deg}^{2}$ and spectra for roughly $93 \times 10^{4}$ galaxies and $12 \times 10^{4}$ QSOs, observed by $2.5 \mathrm{~m}$ telescope at the Apache Point Observa-
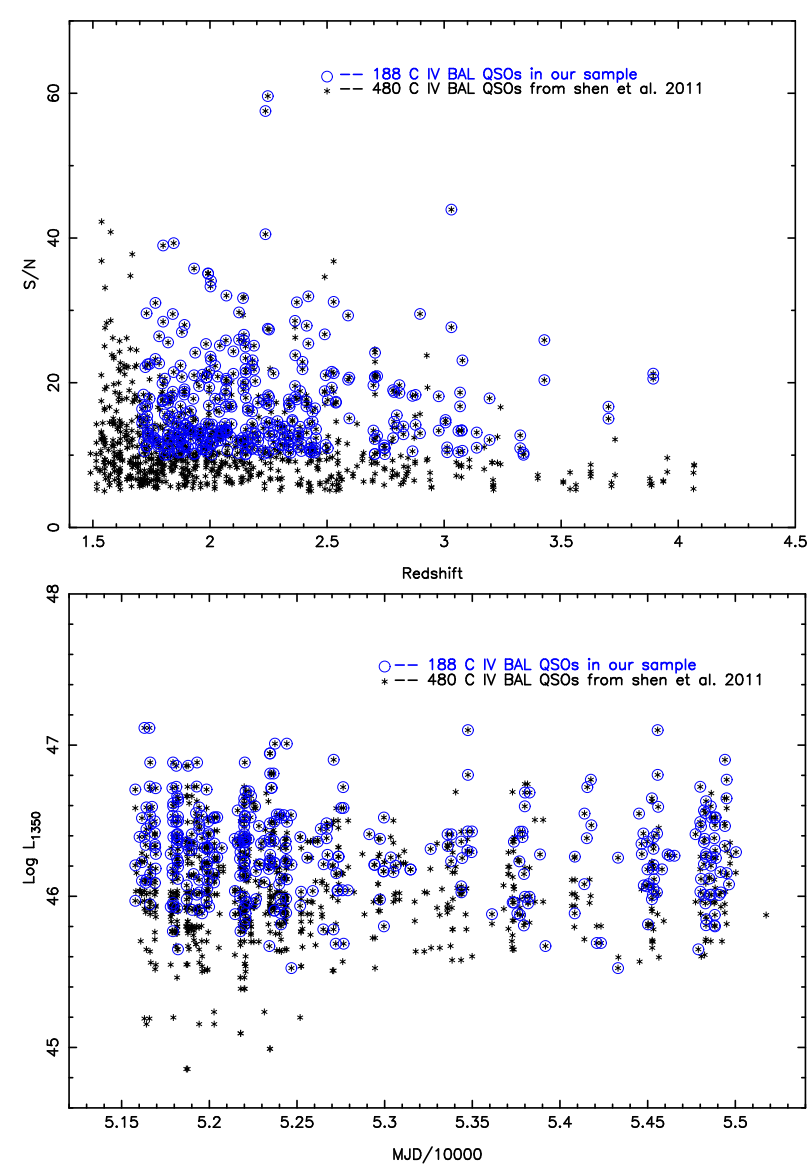

Figure 1. Top: the $\mathrm{S} / \mathrm{N}$ at r-band versus the redshift. Bottom: the continuum luminosity at $1350 \AA\left(L_{1350}\right.$, in units of $\left.\mathrm{erg} \mathrm{s}^{-1}\right)$ versus the MJD. The black stars denote $480 \mathrm{BAL}$ QSOs with at least two-epoch spectra from SDSS DR7. The blue circles denote 188 BAL QSOs with longest time-scale two-epoch spectra with $\mathrm{S} / \mathrm{N}(\mathrm{r})>10$ and $\mathrm{z}>1.7$.

tory in New Mexico. The SDSS spectra were obtained through 3" fibers. For the SDSS DR7 spectra, the observational wavelength coverage is from $3800 \AA$ to $9200 \AA$, and the spectral resolution is 1850 - 2200. With SDSS DR7, Shen et al. (2011) gave a compilation of properties of 105783 QSOs. With the modified balnicity index $\mathrm{BI}_{0}\left(0 \mathrm{~km} \mathrm{~s}^{-1}\right.$ as a minimum detection limit for the outflow velocity range), there are 6214 BAL QSOs identified in their SDSS DR7 sample. It is found that there are 1080 spectra of $480 \mathrm{C}$ IV BAL QSOs with at least two-epoch observations. In order to identify the variable regions in C IV BAL troughs, we select BAL QSOs with SDSS spectral signal-to-noise ratio $(\mathrm{S} / \mathrm{N})$ at $\mathrm{r}$ band larger than 10, and redshift more than 1.7. It is consist of $188 \mathrm{C}$ IV BAL QSOs with 428 SDSS spectra. For each QSO with more than two epochs, we consider only the longest time-scale between two epochs. In Fig. 1 we give the $\mathrm{S} / \mathrm{N}$ versus the redshift, and the luminosity at $1350 \AA$ ( $\left(L_{1350}\right)$ versus the spectral MJD values. The stars denote 480 C IV BAL QSOs with at least two-epoch observations, and the blue circles denote our $188 \mathrm{C}$ IV BAL QSOs with longest twoepoch SDSS spectra with $\mathrm{S} / \mathrm{N}(\mathrm{r})>10$ and $\mathrm{z}>1.7$. In Fig. 11 for our sample of 188 BAL QSOs, the redshift distribution is between 1.7 and about 3.9, the $\mathrm{S} / \mathrm{N}$ distribution is between 10 and about 60 . Considering the problem of spectrophotometric flux calibration for BOSS in SDSS III, we just use the spectra from SDSS DR7 (e.g. Paris et al. 2014; Margala et al. 2015). Our sample is different to 


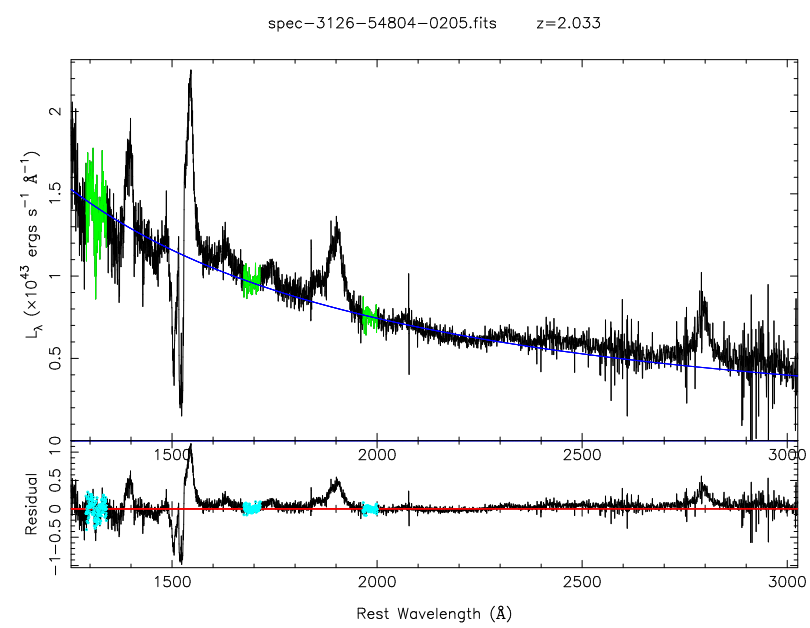

Figure 2. An example of the power-law continuum fit. Top: the extinctioncorrected rest-frame spectrum is shown as the black line. The green dots are the initial continuum windows. The blue line is the power-law continuum. Bottom: the residual spectrum.

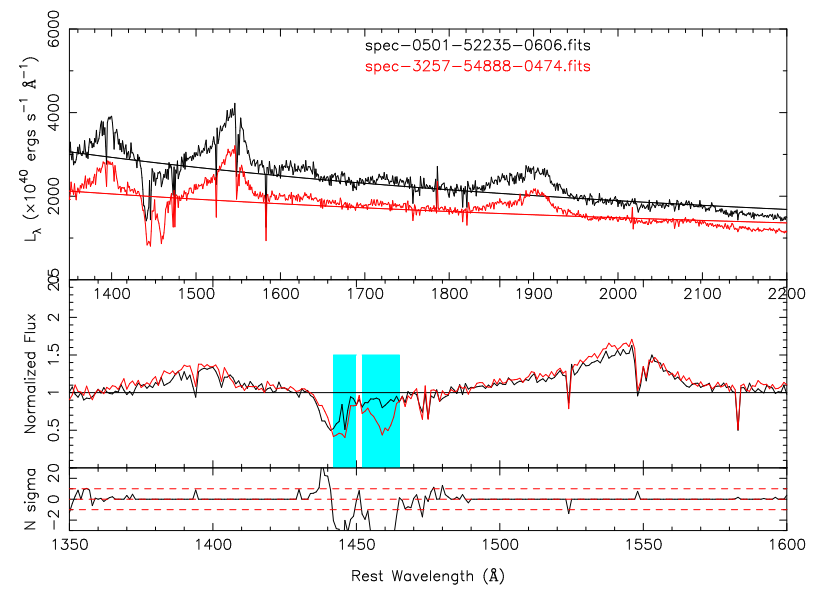

Figure 3. An example of the C IV BAL-trough variable regions. Top: twoepoch spectra as well as their continuums. Middle: their normalized spectra and the variable regions in C IV BAL troughs (cyan shaded regions). Bottom: the $N_{\sigma}$ versus the wavelength $\lambda$. Variable regions of C IV BAL troughs are identified to be where an absorption feature is detected with $\left|N_{\sigma}\right| \geq 1$ for at least five consecutive data points ( $4 \AA$ wide).

that by Filiz Ak et al. (2013), who used two-epoch spectra from the SDSS and the BOSS respectively.

\section{SPECTRAL ANALYSIS}

\subsection{Fitting the spectral continuum}

In order to characterize the spectral variation of BAL QSOs and the variable regions in the $\mathrm{C}$ IV BAL troughs, we fit the continuum spectrum by a power-law function iteratively (He et al. 2014). All the SDSS spectra are corrected for Galactic extinction, assuming the extinction curve of Cardelli et al. (1989) (IR band; UV band) and O'Donnell (1994) (optical band) with $R_{V}=3.1$. The $A_{V}$ values of these SDSS BAL QSOs are derived from the SpecPhotoAll table in SDSS. All spectra are then corrected to the rest frame, and rebinned to a uniform grid with $1 \AA$ wide. It is popularly accepted that we can use one power-law function $f_{\lambda} \propto \lambda^{\alpha}\left(f_{\nu} \propto \nu^{-(2+\alpha)}\right)$ to fit the QSO continuum. Some authors also used two power-law functions to model the QSO continuum (e.g. Forster et al. 2001; Shang et al. 2005). For BAL QSOs, a polynomial function or a reddened power law was also used to fit the continuum in previous studies (Lundgren et al. 2007; Gibson et al. 2008, 2009). Here, we use one power-law function, $f_{\lambda}=f_{2000}(\lambda / 2000)^{\alpha}$, to fit the continuum spectra of BAL QSOs (Hu et al. 2008; He et al. 2014). The power-law continuum is fit iteratively in the "continuum windows", which are known to be relatively free from strong emission lines. Our adopted continuum windows are 12901330, 1685-1715, 1970-2010 $\AA$ in the rest frame (Fig. 2 e.g. Forster et al. 2001; Vanden Berk et al. 2001; Gibson et al. 2008; Bian et al. 2012a; Baskin \& Laor 2013). The fit is achieved by minimizing $\chi^{2}$ iteratively. At each iteration, we neglect any spectral bins that deviate by more than $3 \sigma$ from the previous continuum fit. This iterative method would automatically adjust the fitting window, excluding spectral regions that contain broad emission or absorption features, especially for BAL QSOs.

For the power-law continuum, $f_{\lambda}=f_{2000}(\lambda / 2000)^{\alpha}$, the error of the power-law continuum by error propagation is calculated as follows (He et al. 2014):

$\delta\left(f_{\text {con }}\right)=f_{\lambda} \sqrt{\left(\frac{\delta\left(f_{2000}\right)}{f_{2000}}\right)^{2}+(\ln \lambda-\ln 2000)^{2} \delta \alpha^{2}}$.

where the errors $\left(\delta\left(f_{2000}\right)\right.$ and $\left.\delta \alpha\right)$ are given in the power-law fitting. The total C IV BAL-trough EW is calculated as follows:

$E W=\int\left[1-\frac{f_{o b v}(\lambda)}{f_{\text {con }}(\lambda)}\right] d \lambda$.

The integration is integrated from the total C IV BAL-trough for $f_{o b v}(\lambda)<f_{c o n}(\lambda)$. And the error for the C IV BAL EW is measured as follows:

$\delta(E W)=\sqrt{\sum_{\lambda}\left(\frac{f_{o b v}}{f_{c o n}}\right)^{2}\left[\left(\frac{\delta\left(f_{o b v}\right)}{f_{o b v}}\right)^{2}+\left(\frac{\delta\left(f_{c o n}\right)}{f_{c o n}}\right)^{2}\right]}$.

where $\delta\left(f_{o b v}\right)$ at $\lambda$ is the flux error for the SDSS spectrum.

With the continuum fit, we can obtain the spectral index $\alpha$, the continuum luminosity at $1500 \AA$, and the total C IV BAL EW, as well as their errors. These results are listed in Table 2 An example of the continuum fit and the residual is shown in Fig. 1.

\subsection{Measuring the variable regions in C IV BAL troughs}

In the C IV BAL tough for each QSO, it is possible to have some variable regions. To identify the variable regions in the $\mathrm{C}$ IV BALtrough, we compare two-epoch spectra for BAL QSOs, measuring the flux deviation between two observations at each wavelength by the following equation (Filiz Ak et al. 2013):

$N_{\sigma}(\lambda)=\frac{f_{1}-f_{2}}{\sqrt{\sigma_{1}^{2}+\sigma_{2}^{2}}}$

where $f_{1}$ and $f_{2}$ are the normalized flux based on the fitting powerlaw continuum and $\sigma_{1}$ and $\sigma_{2}$ are the normalized flux error at wavelength $\lambda$. Both $\sigma_{1}$ and $\sigma_{2}$ include observational flux errors and uncertainties on the continuum model. Similar to Gibson et al. (2008), variable regions of BAL troughs are identified to be where an absorption feature is detected with $\left|N_{\sigma}\right| \geq 1$ for at least five consecutive data points ( $4 \AA$ wide). This requirement allows detection of variable regions wider than $774 \mathrm{~km} \mathrm{~s}^{-1}$. It is slightly smaller than 
that by Gibson et al. (2008), and larger than that by Filiz Ak et al. (2013). For the number of data points to be lager than 5, the significance of variations would be $>99.9 \%$. 73 variable regions in the C IV BAL troughs are identified from two-epoch different spectra in 43 BAL QSOs. For the sample of 188 BAL QSOs, there are about 23\% (43/188) BAL QSOs showing variable regions from two-epoch spectra. Fig. 3 gives an example of identified two variable regions in C IV BAL trough.

For each spectrum, we calculate EW and its error for each $\mathrm{C}$ IV BAL variable region (see above Eq. 2. Eq. 3). Then, we derive the EW variance for each C IV BAL variable region for each QSO. The sum of the EW variance for all variable regions in a QSO is adopted as its total EW variance, $\Delta E W$. The error of $\Delta E W$ is calculated based on the error propagation from errors of EW for all variable regions. For each variable region in C IV BAL troughs, the velocity width and the center outflow velocity are also calculated, as well as the maximum outflow velocity $V_{\max }$ of variable regions. The velocity width is calculated from the left and right boundary of the variable region. In Table 3 , we list the total $\Delta E W$, the left and right boundary of the variable region for these 43 BAL QSOs.

\section{RESULTS AND DISCUSSION}

\subsection{Features of the variable regions: velocity width, outflow velocity, variation proportion}

With the above criterion of at least five consecutive data points larger than $1 \sigma, 73$ variable regions in the C IV BAL troughs are identified from 43 two-epoch different spectra. Fig. 4 shows histograms of their velocity width and their center outflow velocity (Table 3. BAL-troughs variation tends to occur on small velocity width. Even the largest variation widths $\left(6500 \mathrm{~km} \mathrm{~s}^{-1}\right)$ are narrow compared to BAL-trough outflow velocity (bottom panel of Fig.4. The number of the C IV BAL variable regions decreases with the increase of the velocity width of the variable regions. These results are consistent with that by Gibson et al. (2008).

From the bottom panel of Fig. 4 variable regions are found across a wide range of central outflow velocities, and the number of variable regions appears to peak in the range between 5000 and $21000 \mathrm{~km} \mathrm{~s}^{-1}$. Considering $\mathrm{BI}_{0}$ used by Shen et al. (2011), $0 \mathrm{~km} \mathrm{~s}^{-1}$ is adopted as a minimum detection limit for BAL-trough. We do not consider contaminations from C IV 1549, Si IV 1399 emission lines at small outflow velocities and large outflow velocities, respectively. It could explain decrease of number of variable regions at small outflow velocities and at large outflow velocities in the bottom panel in Fig. 4

In Fig. 5 we give the distribution of the time-interval between epochs for total 188 BAL QSOs (top panel), as well as the time-interval histogram for $43 \mathrm{BAL}$ QSOs showing variable regions (middle panel). It covers a time-interval of about $0.001-3$ years in the rest frame. The bottom panel in Fig. 5 is the number ratio of the middle panel to the top panel, i.e., the proportion of BAL QSOs showing variable regions versus the time-interval. It is clear that the proportion of BAL QSOs showing variable regions increases with the time-interval, rising to $50 \%$ when the time-interval is longer than about 1 year in the rest frame. This result gives a good reason to consider only the longest time-interval spectral pairs for each QSO. Larger proportion of BAL trough showing variable region across longer time-interval is consistent with the result by Capellupo et al. (2013).
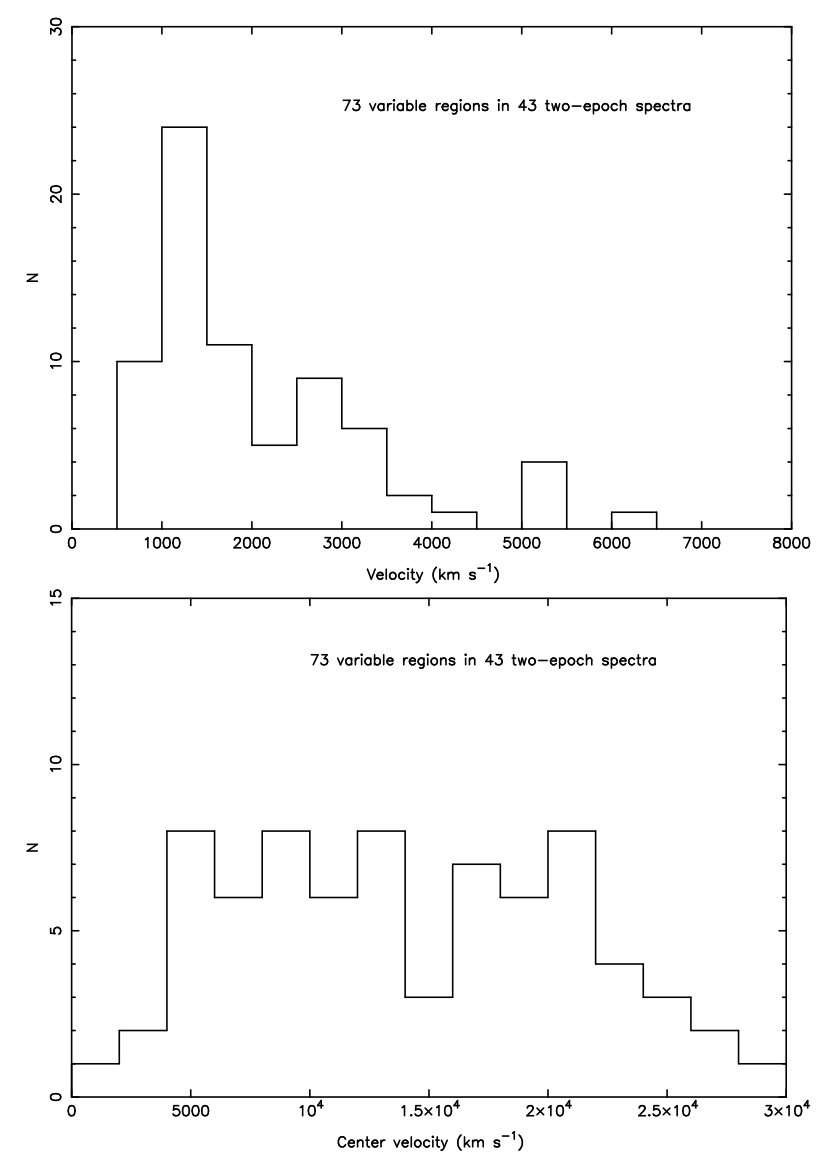

Figure 4. Histograms for the 73 identified variable C IV BAL regions. Top: the distribution of the velocity width of the variable regions. Bottom: the distribution of the center outflow velocity of the variable regions.

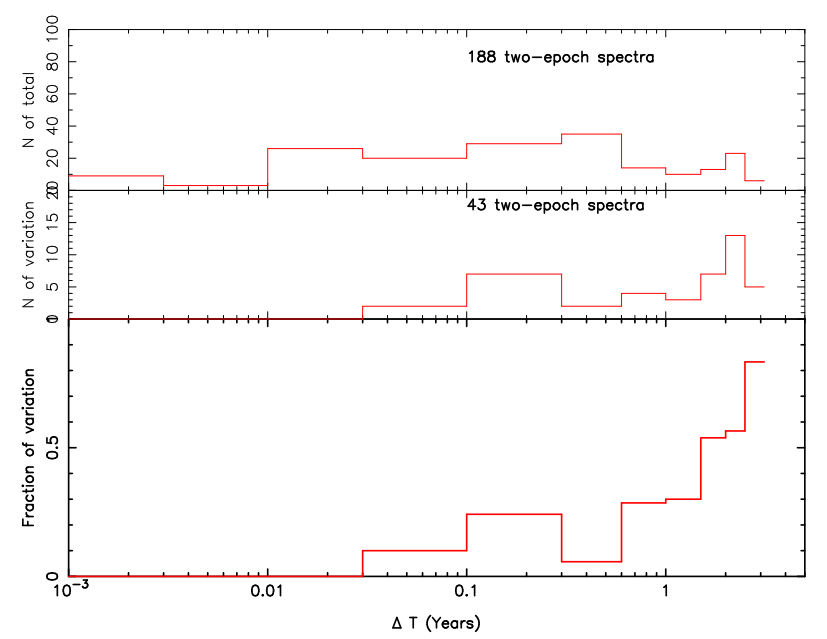

Figure 5. Top: distribution of the time-interval between two epochs for all 188 BAL QSOs. Middle: distribution of the time-interval between two epochs for 43 C IV BAL QSOs with variable regions. Bottom: the number ratio of the middle panel to the top panel. The time-interval is in the rest frame of BAL QSOs. 
Table 1. Summary of the Spearman correlation coefficients: For total sample of 188 BAL QSOs, $\triangle E W$ is the BAL-trough EW variation from two-epoch spectra, and there are $116 \mathrm{BAL}$ QSOs with available Mg II -based $M_{\mathrm{BH}}$. For $43 \mathrm{BAL}$ QSOs with variable regions, $\Delta E W$ is the EW variation of the C IV BAL-trough variable regions, and there are $28 \mathrm{BAL}$ QSOs with available $\mathrm{Mg}$ II -based $M_{\mathrm{BH}} \cdot \Delta L_{1500}$ is the variation of the continuum at $1500 \AA . \Delta \alpha$ is the variation of the spectral index. $M_{\mathrm{BH}}$ is the Mg II -based SMBH masses from a single spectrum. $L_{b o l} / L_{E d d}$ is the ratio of the bolometric luminosity to the Eddington luminosity. $V_{\max }$ is the maximum velocity of the variable region. The value in brackets is the probability of the null hypothesis.

\begin{tabular}{lccccc}
\hline & $\Delta L_{1500}-\Delta \alpha$ & $\Delta E W-\Delta \alpha$ & $\Delta E W-\Delta L_{1500}$ & $\Delta E W-M_{B H}$ & $V_{\text {max }}-L_{\text {bol }} / L_{E d d}$ \\
\hline Total sample & $-0.36(5.5 E-07)$ & $-0.008(0.91)$ & $-0.17(0.02)$ & $-0.15(0.1)$ & - \\
Subsample & $-0.56(8.3 E-05)$ & $0.30(0.05)$ & $-0.44(0.003)$ & $-0.54(0.003)$ & $0.53(0.004)$ \\
\hline
\end{tabular}

\subsection{The variability of spectral index $\Delta \alpha$ for BAL QSOs with variable regions}

It has been shown that QSOs spectra are bluer during their brighter phases. It is usually explained by the variation of the accretion disk. Fig. 6 shows $\Delta \alpha$ versus the continuum variation at $1500 \AA$ ( $\left.\Delta L_{1500}\right)$ for total 188 BAL QSOs (the top panel). There is a significant weak correlation between them. The Spearman coefficient $R$ is -0.36 , with the the probability of the null hypothesis of $P_{\text {null }}=5.5 \times 10^{-7}$ (see Table 11. In the second and fourth quadrants in the top panel in Fig.6 BAL QSOs show bluer during their brighter phase. There are about $56.9 \%$ date points showing bluer during their brighter phase. It is consistent with the result for non-BAL QSOs by Bian et al. (2012a). Considering 11 points with both $\Delta L_{1500}$ and $\Delta \alpha$ more than $3 \sigma$, the proportion rises to $84.6 \%$ (11/13), more QSOs appear bluer during their brighter phases compared to non-BAL QSOs (the bottom panel in Fig. 6.

For the subsample of BAL QSOs with variable regions in C IV BAL troughs, there is a significant medium strong correlation between the $\Delta L_{1500}$ and $\Delta \alpha$. The Spearman coefficient $R$ is -0.56 , $P_{\text {null }}=8.3 \times 10^{-5}$ (see Table 1 ). There are about $76.7 \% \mathrm{BAL}$ QSOs showing bluer during their brighter phases for 43 BAL QSOs (red circles in the top panel in Fig. 6. Considering 6 BAL QSOs with both $\Delta L_{1500}$ and $\Delta \alpha$ more than $3 \sigma$, the proportion of the points in the second and fourth quadrants rises to $100 \%(6 / 6)$ (red circles in bottom panel in Fig. 6).

The proportion showing bluer during the brighter phases rises from about $56.9 \%$ for total 188 BAL QSOs to about $76.7 \%$ for 43 BAL QSOs with variable regions. During the brighter phases, the accretion disk becomes hotter and its emission peak would move to shorter wavelengths. It may lead to the trend of bluer spectra during brighter phases. Therefore, this larger proportion implies that the origin of variable BAL-trough regions is related to the central accretion process.

\subsection{The $\mathrm{EW}$ variability $\Delta E W$ for the variable region: the relation with $\Delta \alpha, \Delta L_{1500}$}

For the subsample of 43 BAL QSOs with variable regions in C IV BAL troughs, the relation between $\Delta E W$ and $\Delta \alpha, \Delta L_{1500}$ are used to investigate the origin of BAL variability (see Table 2, Table 3]. Fig. 7 shows $\Delta E W$ versus $\Delta \alpha$ (top panel). It is possible that there is a weak correlation between $\Delta E W$ and $\Delta \alpha$, according to a Spearman rank correlation test $\left(R=0.3, P_{\text {null }}=0.05\right.$; Table 11. There are $60.5 \%$ of QSOs located in the first and third quadrants where the BAL-troughs become stronger when BAL QSOs become redder. This is different from our previous study for a single BAL QSOs with 18 epochs (He et al. 2014). He et al. (2014) found a strong correlation between $\mathrm{EW}$ variation for C IV BALtrough and $\Delta \alpha(\mathrm{R}=0.77)$, suggesting that dust is intrinsic to outflows. For the subsample of BAL QSOs with variable regions, we

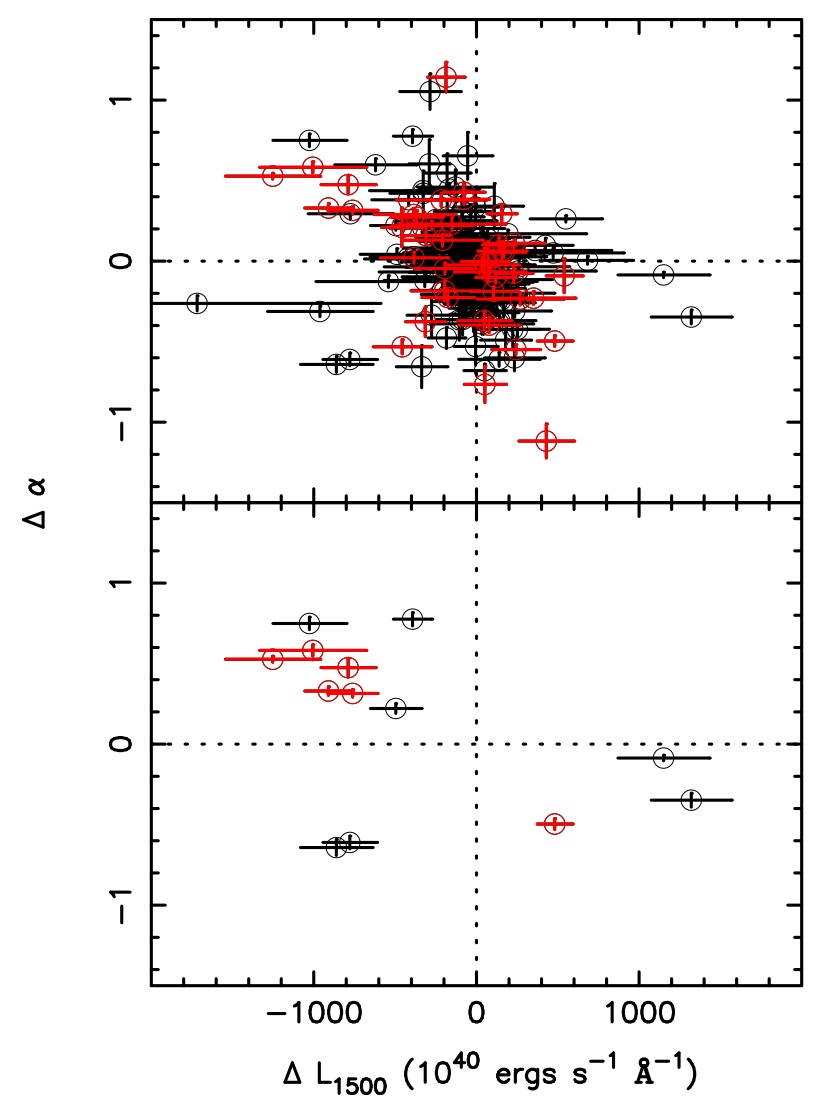

Figure 6. $\Delta \alpha$ versus $\Delta L_{1500}$ for $188 \mathrm{BAL}$ QSOs (top), and for $13 \mathrm{BAL}$ QSOs with both $\Delta L_{1500}$ and $\Delta \alpha$ more than $3 \sigma$ (bottom). The proportion of the points in the second and fourth quadrants (i.e., bluer during brighter phases) rises from about $56.9 \%$ (top) to $84.6 \%$ (bottom). The red circles denote 43 BAL QSOs with variable regions (top), and 6 QSOs with both $\Delta L_{1500}$ and $\Delta \alpha$ more than $3 \sigma$ (bottom). The proportion of the points in the second and fourth quadrants is $76.7 \%$. For 6 QSOs with both $\Delta L_{1500}$ and $\Delta \alpha$ more than $3 \sigma$, the proportion of the points in the second and fourth quadrants rises to $100 \%$.

don't confirm that strong correlation from the two-epoch variation. It is possibly due to the additional parameters to blur this correlation. For the total sample of $188 \mathrm{BAL}$ QSOs, there is no correlation between $\Delta E W$ and $\Delta \alpha$, where $\Delta E W$ is the EW variation for C IV BAL-trough. A Spearman rank correlation test gives $R=-0.008, P_{\text {null }}=0.91$ (Table 1 ).

The bottom panel in Fig. 7 shows $\Delta E W$ versus $\Delta L_{1500}$ for 43 BAL QSOs with variable regions. It is possible that there is a medium strong correlation between them, $R=-0.44, P_{\text {null }}=$ 0.003 (Table 1). It is found that $60.5 \%$ QSOs located in the second and fourth quadrants, where BAL troughs become weaker 

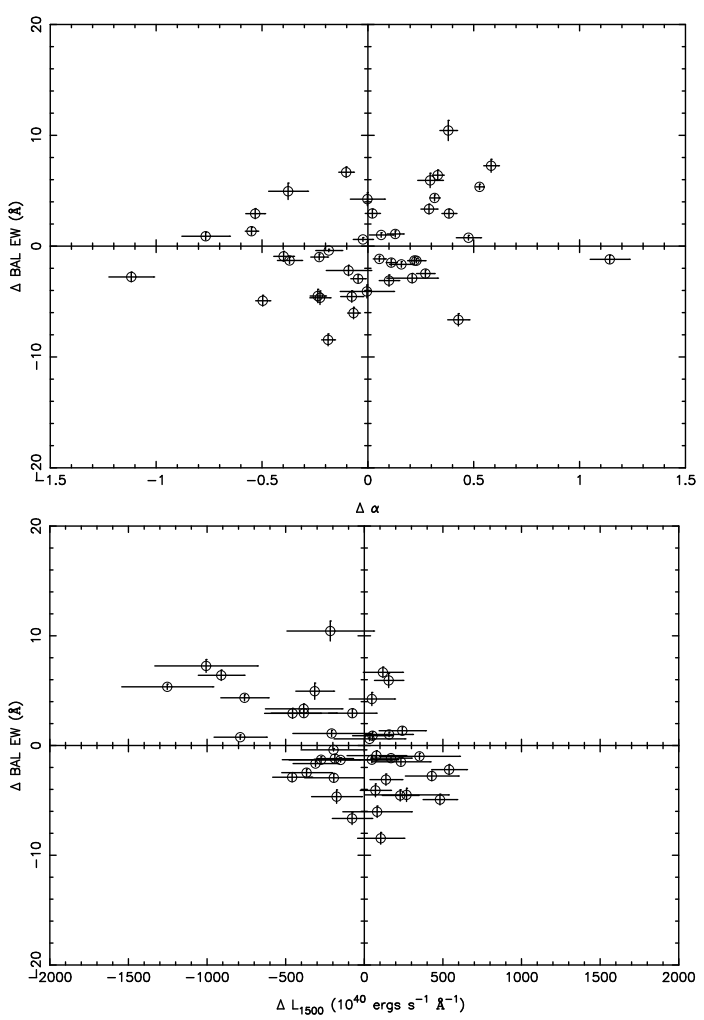

Figure 7. Top: $\Delta E W$ versus $\Delta \alpha$ for 43 epochs in BAL QSOs with variable regions. The Spearman correlation coefficient $R$ is 0.3 with $P_{\text {null }}=0.05$. Bottom: $\Delta E W$ versus $\Delta L_{5100}$ for 43 epochs in BAL QSOs with variable regions, $R=-0.44, P_{\text {null }}=0.003$.

when BAL QSOs become brighter. For the total sample of 188 BAL QSOs, $\triangle E W$ is the EW variation for C IV BAL-trough, and $R=-0.17, P_{\text {null }}=0.02$ (Table 1). Therefore, we don't find a significant correlation between them. It is consistent with the study of Filiz Ak et al. (2013), who found no significant evidence for EW variability of the C IV BAL trough driven by QSO bolometric luminosity (also see Gibson et al. 2008). With respect to total sample of 188 BAL QSOs, the subsample of 43 BAL QSOs with variable regions has a stronger correlation between them. Considering the probability of the null hypothesis $\left(P_{\text {null }}=0.003\right)$ for the subsample of 43 BAL QSOs with variable regions, this correlation is suggestive and requires further investigations by larger sample.

\subsection{The relation of $\Delta E W$ with the central accretion properties, $M_{\mathrm{BH}}, L_{\mathrm{bol}} / L_{\mathrm{Edd}}$}

The relation between the BAL properties/variability and the accretion process in BAL QSOs has been discussed by many authors (e.g. Laor \& Brandt 2002; Ganguly et al. 2007; Baskin \& Laor 2013; Filiz Ak et al. 2013; He et al. 2014). There are two fundamental parameters related to the accretion process, the SMBH mass $\left(M_{\mathrm{BH}}\right)$ and the Eddington ratio $\left(L_{\mathrm{bol}} / L_{\mathrm{Edd}}\right)$. Considering that the C IV -based SMBH mass is biased to the possible nonvirialized component in C IV emission lines (e.g. Rafiee \& Hall 2011; Shen et al. 2011; Bian et al. 2012b), we used Mg II -based $M_{\mathrm{BH}}$ to investigate the relation of $\Delta E W$ with the central accretion properties, such as $M_{\mathrm{BH}}$, and $L_{\mathrm{bol}} / L_{\mathrm{Edd}}$. Fitting $\mathrm{Mg}$ II 2800 emission lines, Shen et al. (2011) gave the Mg II -based $M_{\mathrm{BH}}$ for QSOs with $0.35 \leq z \leq 2.25$. For the subsample of
BAL QSOs with variable regions in C IV BAL toughs, there are 28 BAL QSOs with measured Mg II -based $M_{\mathrm{BH}}$ by Shen et al. (2011). The bolometric luminosity $\left(L_{\mathrm{bol}}\right)$ is calculated from $L_{3000}$ $(0.7 \leq z \leq 1.9), L_{1350}(z \geq 1.9)$. With the bolometric corrections (BC) from the luminosity at $3000 \AA$ and $1350 \AA$ as $B C_{3000}=5.15, B C_{1350}=3.81$, we calculate $L_{\mathrm{bol}} / L_{\mathrm{Edd}}$. These results are listed in Table 3 .

In Fig. 8, we show the relation between $|\Delta E W|$ and $\mathrm{Mg}$ II -based $M_{\mathrm{BH}}$. It is possible that there is a negative medium strong correlation between the $|\Delta E W|$ and $M_{\mathrm{BH}}\left(R=-0.54, P_{\text {null }}=\right.$ 0.003 , Table 11, showing smaller variation of BAL-trough for BAL QSOs with larger SMBHs masses. In Fig. 9, we shows the relation between the maximum outflow velocity of variable regions $\left(V_{\max }\right)$ and the Eddington ratio $L_{\mathrm{bol}} / L_{\mathrm{Edd}}$. It is possible that there is a medium strong correlation between the $V_{\max }$ and $L_{\mathrm{bol}} / L_{\mathrm{Edd}}$ ( $R=0.53, P_{\text {null }}=0.004$, Table 1 , showing larger outflow velocity for BAL QSOs with larger Eddington ratio. Considering the test confidence less than $99.9 \%$ for the subsample of $43 \mathrm{BAL}$ QSOs, these correlations are suggestive. For the total 188 BAL QSOs, there are 116 BAL QSOs with available Mg II -based $M_{\mathrm{BH}}$ , and there is no significant correlation between $|\Delta E W|$ and $M_{\mathrm{BH}}$ $\left(R=-0.15, P_{\text {null }}=0.1\right.$, Table 1 .

In Figs. 8, 9, there is an outlier, SDSS J020006.31-003709.7 ( $z=2.141)$, with largest $M_{\mathrm{BH}}$ and smallest $L_{\mathrm{bol}} / L_{\mathrm{Edd}}$ in Table 3 Excluding this QSO, these correlations would be stronger and significant. For the relation between $|\Delta E W|$ and $M_{\mathrm{BH}}$, $R=-0.7, P_{\text {null }}=0.00005$. For the relation between $V_{\max }$ and $L_{\text {bol }} / L_{\text {Edd }}, R=0.58, P_{\text {null }}=0.0014$. These results imply the connection between the BAL-trough variation and the central accretion process. Using $\mathrm{C}$ IV -based SMBH mass for BAL QSOs $(z>2)$, Filiz Ak et al. (2013) suggested possible correlations between $|\Delta E W|$ and luminosity/Eddington ratio for $\mathrm{C}$ IV troughs on moderate time-scales (1-2.5 yrs). They did not find significant correlation between $|\Delta E W|$ and $M_{\mathrm{BH}}$. These correlations require further investigations in larger samples of BAL QSOs with no bias $M_{\mathrm{BH}}$ estimation such as from $\mathrm{H} \beta, \mathrm{Mg}$ II emission lines.

We don't find a significant correlation between $V_{\max }$ and $\alpha$, $L_{\text {bol }}$. We don't confirm the result by Laor \& Brandt (2002), who found that $V_{\max }$ increase with $L_{\mathrm{bol}}$. We find that $L_{\mathrm{bol}} / L_{\mathrm{Edd}}$ instead of $L_{\mathrm{bol}}$ is a driver of $V_{\max }$. In the future, we can use the BOSS spectra to estimate the $M_{\mathrm{BH}}$ because of their larger wavelength coverage in BOSS spectra than in SDSS spectra.

\section{SUMMARY}

The variability of broad absorption lines is investigated for a sample of 188 BAL QSOs $(z>1.7)$ with at least two-epoch observations from the SDSS DR7. Considering only the longest time-scale between epochs for each QSO, 73 variable regions in the $\mathrm{C}$ IV BAL troughs are detected for 43 BAL QSOs. The main conclusions can be summarized as follows.

(1) Considering the flux deviation $\left|N_{\sigma}\right| \geq 1$ for at least five consecutive data points ( $4 \AA$ wide) in the two-epoch difference spectrum, $73 \mathrm{C}$ IV BAL variable regions in 43 BAL QSOs are identified from 188 BAL QSOs, i.e., about 23\% (43/188) BAL QSOs showing variable regions from two-epoch spectra. It is found that the proportion of BAL QSOs showing variable regions increases with the time-interval longer than about 1 year in the rest frame. BAL variation tends to occur on small velocity width. Even the largest variation widths $\left(6500 \mathrm{~km} \mathrm{~s}^{-1}\right)$ are narrow compared to BAL-trough outflow velocity. Variable regions are found across a 


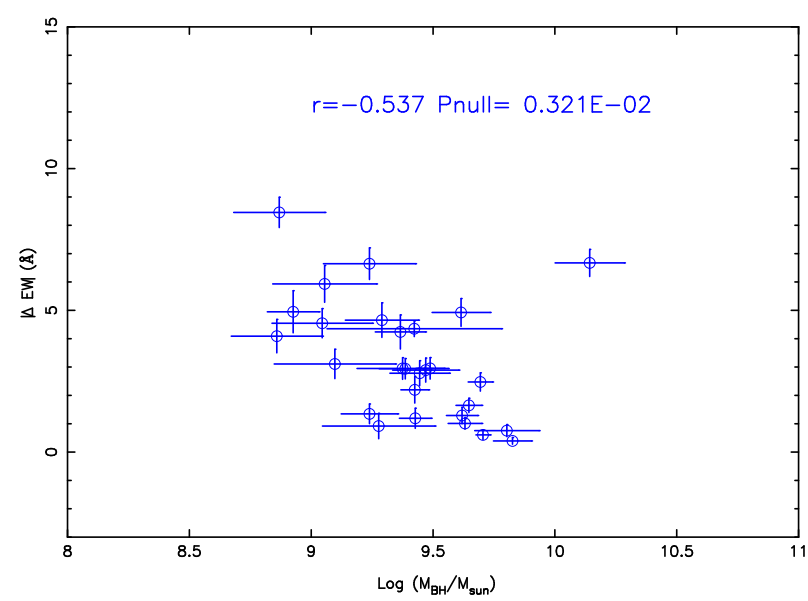

Figure 8. $|\Delta E W|$ versus the $\mathrm{Mg}$ II -based $M_{\mathrm{BH}}$ for $28 \mathrm{BAL}$ QSOs with variable regions. The Spearman correlation coefficient $R$ is -0.54 with $P_{\text {null }}=0.003$.

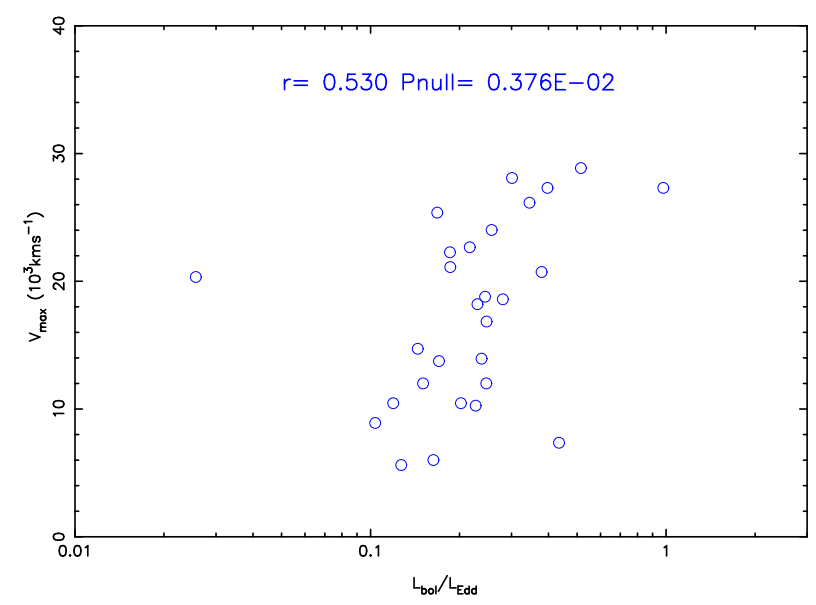

Figure 9. The maximum outflow velocity of variable regions $\left(V_{\max }\right)$ versus the Eddington ratio $L_{\mathrm{bol}} / L_{\mathrm{Edd}}$ for $28 \mathrm{BAL}$ QSOs with variable regions. The Spearman correlation coefficient $R$ is 0.53 with $P_{\text {null }}=0.004$.

wide range of outflow velocities, and the number of variable regions appears to peak in the range between $\sim 5000$ and $\sim 21000$ $\mathrm{km} \mathrm{s}^{-1}$.

(2) With two-epoch variation, it is found that there is a weak correlation between $\Delta L_{1500}$ and $\Delta \alpha$ for total 188 two-epoch spectra, and about half BAL QSOs appear redder during their brighter phases. It is consistent with the result for non-BAL QSOs by (Bian et al. 2012a). For a subsample of BAL QSOs with variable regions in BAL toughs, their correlation becomes stronger, about 76.7\% BAL QSOs appear bluer during their brighter phases. This larger proportion implies that the origin of variable regions in BALtrough is related to the central accretion process.

(3) For the subsample of 43 BAL QSOs with variable regions, it is possible that there is a weak correlation between $\triangle E W$ and $\Delta \alpha$. It is different from our previous study for a single BAL QSOs by (He et al. 2014). It is possibly due to the additional parameters to blur this correlation. It is possible that there is a medium strong correlation between $\Delta E W$ and $\Delta L_{5100}$. Considering the test confidence is $99.7 \%$, this latter correlation is suggestive and requires further investigations by larger sample.

(4) For the subsample of 43 BAL QSOs with variable regions, there are 28 BAL QSOs with available $\mathrm{Mg}$ II -based $M_{\mathrm{BH}}$. It is possible that there is a negative medium strong correlation between the $|\Delta E W|$ and $M_{\mathrm{BH}}$, showing smaller variation of BAL-trough for BAL QSOs with larger SMBHs masses. It is possible that there is a medium strong correlation between $V_{\max }$ of variable regions and $L_{\mathrm{bol}} / L_{\mathrm{Edd}}$, showing larger outflow velocity for BAL QSOs with larger Eddington ratio. These results imply the connection between the BAL-trough variation and the central accretion process. These correlations require further investigations in larger samples of BAL QSOs with no bias $M_{\mathrm{BH}}$ estimation such as from $\mathrm{H} \beta, \mathrm{Mg}$ II emission lines.

\section{ACKNOWLEDGMENTS}

We are very grateful to the anonymous referee for her/his instructive comments which significantly improved the content of the paper. This work has been supported by the National Science Foundations of China (Nos. 11373024, 11173016 and 11233003).

\section{REFERENCES}

Allen J. T., et al. 2011, MNRAS, 410, 860

Baskin A., Laor A., \& Hamann F. 2013, MNRAS, 432, 1525

Bentz M. C., Peterson B. M., Netzer H., Pogge R. W., \& Vestergaard M. 2009, ApJ, 697, 160

Bian W. H., et al., 2012a, ApJ, 759, 88

Bian W. H., et al., 2012b, MNRAS, 427, 2881

Bruni G., et al., 2012, A\&A, 542, 13

Capellupo D. M., et al., 2011, MNRAS, 413, 908

Capellupo D. M., et al., 2012, MNRAS, 422, 3249

Capellupo D. M., et al., 2013, MNRAS, 429, 1872

Cardelli J. A., Clayton G. C., \& Mathis J. S., 1989, ApJ, 345, 245

Elvis M., 2000, ApJ, 545, 63

Fabian A. C., 2012, ARA\&A, 50, 455

Filiz Ak, N., er al., 2012, ApJ, 757, 114

Filiz Ak, N., er al., 2013, ApJ, 777, 168

Fine S., Jarvis M.J., \& Mauch T., 2011, MNRAS, 412, 213

Forster K., et al., 2001, ApJS, 134, 35

Ganguly R., Brotherton M. S., Cales S., et al. 2007, ApJ, 665, 990

Gibson R. R., et al., 2008, ApJ, 675, 985

Gibson R. R., et al., 2009, ApJ, 692, 758

Grier C. J., et al., 2015, ApJ, 806, 111

Guo H. X., \& Gu M. F., 2014, JApA, 35, 477

Hewett P. C., \& Foltz, C. B., Chaffee, F. H., 1995, AJ, 109, 1498H

He Z. C., Bian W. H., Jiang X. L., \& Wang Y. F., 2014, MNRAS, 443, 2532

Hu C., Wang J. M., Ho L. C., Chen Y. M., Zhang H. T., Bian, W. H., Xue, S. J., 2008, ApJ, 687, 78

Kaspi S., Maoz D., Netzer H., et al. 2005, ApJ, 629, 61

Laor A., \& Brandt W. N., 2002, ApJ, 569, 64

Lundgren B. F., Wilhite B. C., Brunner R. J., et al., 2007, ApJ, 656, 73

Margala D, et al., ApJ, arXiv:1506.04790v1

Meusinger H., Hinze, A., de Hoon, A., 2011, A\&A, 525, 37

Montenegro-Montes F. M., et al.,2008, MNRAS, 388, 1853

Murray N., Chiang J., Grossman S. A., \& Voit G. M., 1995, ApJ, 451, 498

O’Donnell J. E., 1994, ApJ, 422, 158

Paris I. et al., 2014, A\&A, 563, 54

Proga D., Stone J. M., \& Kallman T. R., 2000, ApJ, 543, 686 
Pu X. T., Bian W. H., \& Huang K. L., 2006, MNRAS, 372, 24

Rafiee A., \& Hall P. B., 2011, MNRAS, 415, 2932

Shang Z. H., et al., 2005, ApJ, 619, 41

Shen Y., et al., 2011, ApJS, 194, 45

Trump J. R., et al., 2006, ApJS, 165, 1

Urry C. M., \& Padovani P., 1995, PASP, 107, 803

Vanden Berk D. E., Richards G. T., Bauer A., et al., 2001, AJ, 122, 54

Weymann R. J., Morris S. L., Foltz C. B., \& Hewett P. C., 1991, ApJ, 373, 23

Wilhite, B. C., et al., 2005, ApJ, 633, 638

York D. G., Adelman J., Anderson J. E., et al. 2000, AJ, 120, 1579

Zubovas K., \& King, A., 2013, ApJ, 769, 51

Zuo W. W., et al., 2012, ApJ, 758, 104 
BAL variable regions

Table 2. The properties of 188 BAL QSOs. Col. (1) is the No. of BAL QSOs. Col. (2) is No. of the epoch. Col. (3) is the SDSS name. Col. (4) is the redshift. Col. (5) is Plate-MJD-Fiber of the observation. Col. (6) is luminosity in units of $10^{40} \mathrm{erg} \mathrm{s}^{-1} \AA^{-1}$. Col. (7) is the UV spectral index. Col. (8) is the total C IV BAL EW in units of $\AA$.

\begin{tabular}{|c|c|c|c|c|c|c|c|}
\hline $\begin{array}{l}\text { No. } \\
\text { (1) }\end{array}$ & $\begin{array}{l}\text { epoch } \\
\text { (2) }\end{array}$ & $\begin{array}{c}\text { name } \\
(3)\end{array}$ & $\begin{array}{c}\mathrm{z} \\
(4)\end{array}$ & $\begin{array}{l}\text { Plate-MJD-Fiber } \\
\text { (5) }\end{array}$ & $\begin{array}{l}L_{1500} \\
\quad(6)\end{array}$ & $\begin{array}{l}\alpha \\
\text { (7) }\end{array}$ & $\begin{array}{c}\text { BAL EW } \\
\text { (8) }\end{array}$ \\
\hline 001 & 01 & J001130.55+005550.7 & 2.309 & 0389-51795-0339 & $1860.73 \pm 220.18$ & $-1.18 \pm 0.04$ & $12.03 \pm 1.21$ \\
\hline 001 & 02 & J001130.55+005550.7 & 2.309 & 0686-52519-0603 & $1475.93 \pm 154.57$ & $-1.15 \pm 0.03$ & $10.18 \pm 1.10$ \\
\hline 002 & 01 & J001438.28-010750.1 & 1.806 & $0389-51795-0211$ & $770.10 \pm 148.78$ & $-0.88 \pm 0.13$ & $24.11 \pm 2.07$ \\
\hline 002 & 02 & J001438.28-010750.1 & 1.806 & $0687-52518-0249$ & $615.19 \pm 128.45$ & $-0.81 \pm 0.13$ & $21.59 \pm 2.29$ \\
\hline 003 & 01 & J002127.88+010420.1 & 1.820 & $0390-51816-0445$ & $1604.75 \pm 143.90$ & $-1.75 \pm 0.08$ & $14.41 \pm 0.98$ \\
\hline 003 & 02 & J002127.88+010420.1 & 1.820 & 0688-52203-0374 & $1349.17 \pm 129.10$ & $-1.70 \pm 0.08$ & $14.40 \pm 1.04$ \\
\hline 004 & 01 & J002146.71-004847.9 & 2.503 & 0390-51816-0161 & $1824.74 \pm 183.96$ & $-1.22 \pm 0.04$ & $5.35 \pm 1.10$ \\
\hline 004 & 02 & J002146.71-004847.9 & 2.503 & 0390-51900-0180 & $2074.63 \pm 134.06$ & $-1.42 \pm 0.02$ & $4.82 \pm 0.69$ \\
\hline 005 & 01 & J002710.06-094435.3 & 2.070 & 0653-52145-0556 & $1869.96 \pm 126.53$ & $-0.76 \pm 0.03$ & $16.80 \pm 0.75$ \\
\hline 005 & 02 & J002710.06-094435.3 & 2.070 & $3105-54825-0310$ & $1974.66 \pm 75.48$ & $-0.95 \pm 0.01$ & $10.82 \pm 0.41$ \\
\hline 006 & 01 & $\mathrm{~J} 003312.25+155442.4$ & 1.937 & 0417-51821-0576 & $770.90 \pm 88.62$ & $-1.20 \pm 0.12$ & $69.25 \pm 1.26$ \\
\hline 006 & 02 & J003312.25+155442.4 & 1.937 & $3133-54789-0379$ & $841.70 \pm 38.27$ & $-1.21 \pm 0.05$ & $62.26 \pm 0.51$ \\
\hline 007 & 01 & J004118.59+001742.4 & 1.765 & 0392-51793-0631 & $723.13 \pm 113.58$ & $-1.05 \pm 0.10$ & $16.72 \pm 1.55$ \\
\hline 007 & 02 & J004118.59+001742.4 & 1.765 & $0690-52261-0553$ & $712.34 \pm 91.32$ & $-1.38 \pm 0.10$ & $13.40 \pm 1.41$ \\
\hline 008 & 01 & J004323.43-001552.4 & 2.798 & 0393-51794-0181 & $3052.29 \pm 367.63$ & $-0.86 \pm 0.02$ & $9.68 \pm 1.03$ \\
\hline 008 & 02 & J004323.43-001552.4 & 2.798 & $0690-52261-0028$ & $2511.14 \pm 252.10$ & $-0.99 \pm 0.03$ & $12.28 \pm 1.17$ \\
\hline 009 & 01 & $\mathrm{~J} 004527.68+143816.1$ & 1.992 & 0419 & $3911.52 \pm 16$ & $-0.27 \pm 0.03$ & $27.37 \pm 0.52$ \\
\hline 009 & 02 & J004527.68+143816.1 & 1.992 & 0419-51868-0106 & $3944.77 \pm 154.36$ & $-0.30 \pm 0.03$ & $28.71 \pm 0.48$ \\
\hline 010 & 01 & J004613.54+010425.7 & 2.152 & 0393-51794-0572 & $2955.44 \pm 172.22$ & $-1.66 \pm 0.02$ & $39.71 \pm 0.54$ \\
\hline 010 & 02 & $\mathrm{~J} 004613.54+010425.7$ & 2.152 & $0691-52199-0460$ & $2858.19 \pm 131.32$ & $-1.67 \pm 0.02$ & $37.86 \pm 0.42$ \\
\hline 011 & 01 & $\mathrm{~J} 004732.73+002111.3$ & 2.878 & 0393-51794-0588 & $2669.00 \pm 276.06$ & $-1.91 \pm 0.03$ & $8.81 \pm 1.12$ \\
\hline 011 & 02 & J004732.73+002111.3 & 2.878 & 0691-52199-0559 & $2749.06 \pm 207.40$ & $-1.89 \pm 0.02$ & $8.81 \pm 0.83$ \\
\hline 012 & 01 & J004806.05-010321.6 & 2.528 & 0394-51812-0299 & $3502.07 \pm 173.05$ & $-1.73 \pm 0.02$ & $4.79 \pm 0.55$ \\
\hline 012 & 02 & J004806.05-010321.6 & 2.528 & $3111-54800-0281$ & $4051.75 \pm 136.42$ & $-1.47 \pm 0.01$ & $3.70 \pm 0.37$ \\
\hline 013 & 01 & J005419.99+002727.9 & 2.490 & 0394-51812-0511 & $2561.01 \pm 152.81$ & $-1.13 \pm 0.02$ & $16.86 \pm 0.62$ \\
\hline 013 & 02 & J005419.99+002727.9 & 2.490 & $3111-54800-0509$ & $2793.79 \pm 106.36$ & $-1.02 \pm 0.01$ & $13.95 \pm 0.39$ \\
\hline 014 & 01 & J010859.53-105757.8 & 1.807 & 0659-52199-0099 & $1260.76 \pm 105.13$ & $-1.18 \pm 0.07$ & $48.50 \pm 1.02$ \\
\hline 014 & 02 & J010859.53-105757.8 & 1.807 & $3109-54833-0003$ & $945.67 \pm 58.56$ & $-1.56 \pm 0.06$ & $52.70 \pm 0.72$ \\
\hline 015 & 01 & $20.96-100127.0$ & & 2864 & $1277.23 \pm 7$ & $-1.68 \pm$ & $9.86 \pm 0.63$ \\
\hline 015 & 02 & J010920.96-100127.0 & 2.449 & $3109-54833-0080$ & $884.09 \pm 93.49$ & $-0.91 \pm 0.04$ & $9.54 \pm 1.17$ \\
\hline 016 & 01 & J012603.62-100114.8 & 2.302 & 0661-52163-0114 & $1512.19 \pm 160.55$ & $-1.95 \pm 0.03$ & $22.54 \pm 1.06$ \\
\hline 016 & 02 & J012603.62-100114.8 & 2.302 & $2878-54465-0274$ & $1055.95 \pm 77.02$ & $-2.48 \pm 0.03$ & $31.04 \pm 0.79$ \\
\hline 017 & 01 & $\mathrm{~J} 013012.36+153158.0$ & 2.352 & 0425-51898-0445 & $1778.04 \pm 174.69$ & $0.07 \pm 0.03$ & $20.49 \pm 1.06$ \\
\hline 017 & 02 & $\mathrm{~J} 013012.36+153158.0$ & 2.352 & 0425-51884-0446 & $1769.05 \pm 189.22$ & $-0.18 \pm 0.04$ & $23.50 \pm 1.15$ \\
\hline 018 & 01 & J013038.79+391818.1 & 2.520 & $2062-53381-0623$ & $2139.81 \pm 119.40$ & $-1.27 \pm 0.02$ & $13.34 \pm 0.60$ \\
\hline 018 & 02 & J013038.79+391818.1 & 2.520 & $2063-53359-0024$ & $2024.22 \pm 121.10$ & $-1.39 \pm 0.02$ & $12.69 \pm 0.64$ \\
\hline 019 & 01 & J013625.65-103346.2 & 2.009 & $0662-52147-0002$ & $799.65 \pm 121.52$ & $-0.14 \pm 0.08$ & $42.02 \pm 1.68$ \\
\hline 019 & 02 & J013625.65-103346.2 & 2.009 & $1915-53612-0133$ & $650.34 \pm 75.17$ & $-0.27 \pm 0.05$ & $42.34 \pm 1.26$ \\
\hline 020 & 01 & J013656.31-004623.7 & 1.716 & $0400-51820-0003$ & $1338.49 \pm 183.80$ & $-1.10 \pm 0.09$ & $13.55 \pm 1.52$ \\
\hline 020 & 02 & J013656.31-004623.7 & & & & $-0.83 \pm 0.06$ & \\
\hline 021 & 01 & J014948.74+141300.9 & 2.188 & 0429-51820-0584 & $1745.76 \pm 104.38$ & $-1.43 \pm 0.02$ & $7.85 \pm 0.62$ \\
\hline 021 & 02 & J014948.74+141300.9 & 2.188 & $1899-53262-0548$ & $1740.28 \pm 82.23$ & $-1.53 \pm 0.02$ & $7.84 \pm 0.51$ \\
\hline 022 & 01 & $\mathrm{~J} 015048.83+004126.2$ & 3.702 & 0402-51793-0505 & $5558.73 \pm 320.86$ & $-1.30 \pm 0.04$ & $10.18 \pm 0.63$ \\
\hline 022 & 02 & $\mathrm{~J} 015048.83+004126.2$ & 3.702 & 0699-52202-0629 & $5319.60 \pm 315.36$ & $-1.29 \pm 0.05$ & $12.07 \pm 0.68$ \\
\hline 023 & 01 & J020006.31-003709.7 & 2.141 & 0403-51871-0070 & $1846.41 \pm 112.19$ & $-0.41 \pm 0.03$ & $40.36 \pm 0.73$ \\
\hline 023 & 02 & J020006.31-003709.7 & 2.141 & 2866-54478-0197 & $1964.77 \pm 54.49$ & $-0.51 \pm 0.02$ & $48.21 \pm 0.39$ \\
\hline 024 & 01 & J021818.14-092153.5 & 1.880 & $0668-52162-0218$ & $2259.30 \pm 149.56$ & $-1.62 \pm 0.05$ & $16.67 \pm 0.73$ \\
\hline 024 & 02 & J021818.14-092153.5 & 1.880 & $3122-54821-0201$ & $1470.26 \pm 73.36$ & $-1.14 \pm 0.03$ & $19.90 \pm 0.51$ \\
\hline 025 & 01 & J022036.27-081242.9 & 2.004 & $0668-52162-0547$ & $2005.37 \pm 129.03$ & $-0.89 \pm 0.03$ & $20.99 \pm 0.71$ \\
\hline 025 & 02 & J022036.27-081242.9 & 2.004 & & $2247.01 \pm 71.30$ & $-1.44 \pm 0.01$ & $20.66 \pm 0.36$ \\
\hline 026 & 01 & $\mathrm{~J} 022349.24+004727.8$ & & $0704-52205-0459$ & $912.86 \pm 100.24$ & $-1.24 \pm 0.09$ & $13.93 \pm 1.19$ \\
\hline 026 & 02 & $\mathrm{~J} 022349.24+004727.8$ & & & $964.99 \pm 77.56$ & $-2.01 \pm 0.07$ & $19.04 \pm 0.82$ \\
\hline 027 & 01 & $\mathrm{~J} 022844.09+000217.0$ & 2.705 & 0406-51817-0035 & $3900.33 \pm 187.14$ & $-1.02 \pm 0.02$ & $18.87 \pm 0.70$ \\
\hline 027 & 02 & $\mathrm{~J} 022844.09+000217.0$ & 2.705 & 0406-52238-0039 & $3854.36 \pm 202.67$ & $-0.94 \pm 0.02$ & $22.61 \pm 0.73$ \\
\hline 028 & 01 & J023139.53+001758.4 & 2.375 & $0407-51820-0483$ & $1583.17 \pm 118.98$ & $-1.82 \pm 0.03$ & $9.29 \pm 0.87$ \\
\hline 028 & 02 & J023139.53+001758.4 & 2.375 & 0705-52200-0505 & $1372.57 \pm 107.43$ & $-1.82 \pm 0.03$ & $12.73 \pm 0.94$ \\
\hline 029 & 01 & J023252.80-001351.1 & 2.033 & $0705-52200-0063$ & $1231.68 \pm 111.34$ & $-1.95 \pm 0.04$ & $26.64 \pm 1.08$ \\
\hline 029 & 02 & J023252.80-001351.1 & 2.033 & 3126-54804-0205 & $1153.97 \pm 57.90$ & $-1.53 \pm 0.02$ & $17.54 \pm 0.58$ \\
\hline 030 & 01 & J023820.90+001419.7 & 2.782 & 0706-52199-0472 & $2195.28 \pm 223.61$ & $-1.94 \pm 0.03$ & $22.57 \pm 1.28$ \\
\hline 030 & 02 & J023820.90+001419.7 & 2.782 & $3126-54804-0500$ & $1878.07 \pm 115.63$ & $-2.06 \pm 0.02$ & $18.17 \pm 0.72$ \\
\hline
\end{tabular}


Table 2. - continue

\begin{tabular}{|c|c|c|c|c|c|c|c|}
\hline $\begin{array}{l}\text { No. } \\
\text { (1) }\end{array}$ & $\begin{array}{l}\text { epoch } \\
\text { (2) }\end{array}$ & $\begin{array}{l}\text { name } \\
(3)\end{array}$ & $\begin{array}{c}\mathrm{Z} \\
(4)\end{array}$ & $\begin{array}{l}\text { Plate-MJD-Fiber } \\
\qquad(5)\end{array}$ & $\begin{array}{l}L_{1500} \\
\quad(6)\end{array}$ & $\begin{array}{c}\alpha \\
\text { (7) }\end{array}$ & $\begin{array}{l}\text { BAL EW } \\
\text { (8) }\end{array}$ \\
\hline 031 & 01 & J023903.43-003850.8 & 3.075 & 0408-51821-0134 & $2955.93 \pm 219.75$ & $-1.50 \pm 0.03$ & $6.99 \pm 0.98$ \\
\hline 031 & 02 & J023903.43-003850.8 & 3.075 & 0706-52199-0179 & $2807.41 \pm 207.90$ & $-1.61 \pm 0.03$ & $7.91 \pm 0.92$ \\
\hline 032 & 01 & J024221.87+004912.6 & 2.069 & 0408-51821-0576 & $2171.53 \pm 137.44$ & $-1.00 \pm 0.02$ & $19.70 \pm 0.63$ \\
\hline 032 & 02 & J024221.87+004912.6 & 2.069 & 0706-52199-0617 & $2028.23 \pm 147.32$ & $-0.98 \pm 0.03$ & $21.83 \pm 0.71$ \\
\hline 033 & 01 & J024224.02+010452.5 & 2.433 & 0408-51821-0564 & $1394.86 \pm 152.27$ & $-0.61 \pm 0.04$ & $12.18 \pm 1.16$ \\
\hline 033 & 02 & J024224.02+010452.5 & 2.433 & 0706-52199-0606 & $1065.54 \pm 137.63$ & $-0.41 \pm 0.04$ & $11.56 \pm 1.37$ \\
\hline 034 & 01 & J024304.68+000005.4 & 1.995 & 0408-51821-0080 & $1674.30 \pm 135.82$ & $-1.60 \pm 0.03$ & $13.76 \pm 0.83$ \\
\hline 034 & 02 & J024304.68+000005.4 & 1.995 & 0706-52199-0080 & $1464.74 \pm 133.02$ & $-1.78 \pm 0.04$ & $14.05 \pm 1.00$ \\
\hline 035 & 01 & J024701.18+000330.2 & 2.152 & 0409-51871-0472 & $745.43 \pm 118.16$ & $0.11 \pm 0.06$ & $8.28 \pm 1.62$ \\
\hline 035 & 02 & J024701.18+000330.2 & 2.152 & $1664-52973-0474$ & $659.55 \pm 48.46$ & $0.21 \pm 0.03$ & $6.50 \pm 0.79$ \\
\hline 036 & 01 & J025042.45+003536.7 & 2.385 & $0410-51816-0352$ & $1730.23 \pm 157.63$ & $-0.49 \pm 0.03$ & $40.90 \pm 1.02$ \\
\hline 036 & 02 & J025042.45+003536.7 & 2.385 & $1664-52973-0548$ & $951.55 \pm 50.05$ & $-1.10 \pm 0.02$ & $43.20 \pm 0.58$ \\
\hline 037 & 01 & J025331.93+001624.6 & 1.821 & 0410-51816-0391 & $1174.54 \pm 155.96$ & $-2.00 \pm 0.10$ & $9.37 \pm 1.44$ \\
\hline 037 & 02 & J025331.93+001624.6 & 1.821 & 0708-52175-0472 & $1130.18 \pm 95.74$ & $-2.01 \pm 0.07$ & $5.25 \pm 0.95$ \\
\hline 038 & 01 & J031331.22-070422.8 & 2.786 & 0459-51924-0490 & $2468.98 \pm 220.86$ & $-1.85 \pm 0.03$ & $41.52 \pm 1.03$ \\
\hline 038 & 02 & $\mathrm{~J} 031331.22-070422.8$ & 2.786 & $3185-54829-0$ & $1848.56 \pm 120$ & $-1.25 \pm 0.02$ & $42.18 \pm 0.73$ \\
\hline 039 & 01 & J031609.83+004043.1 & 2.897 & 0413-51821-0386 & $3229.25 \pm 401$ & $-1.16 \pm 0.03$ & $9.04 \pm 1.19$ \\
\hline 039 & 02 & J031609.83+004043.1 & 2.897 & $3183-54833-0465$ & $3258.12 \pm 164.31$ & $-1.28 \pm 0.01$ & $8.83 \pm 0.46$ \\
\hline 040 & 01 & J031828.90-001523.1 & 1.985 & 0413-51821-0166 & $3089.15 \pm 166.78$ & $-1.82 \pm 0.02$ & $7.94 \pm 0.59$ \\
\hline 040 & 02 & J031828.90-001523.1 & 1.985 & 0711-52202-0114 & $2600.48 \pm 154.31$ & $-1.78 \pm 0.03$ & $10.64 \pm 0.65$ \\
\hline 041 & 01 & J032118.22-010539.9 & 2.412 & $0413-5$ & $2625.51 \pm 193.52$ & $-0.12 \pm 0.03$ & $23.14 \pm 0.85$ \\
\hline 041 & 02 & J032118.22-010539.9 & 2.412 & 0712-52199-0287 & $1598.91 \pm 115.59$ & $0.63 \pm 0.03$ & $18.29 \pm 0.87$ \\
\hline 042 & 01 & J032701.43-002207.1 & 2.319 & 0414-51869-0152 & $1666.84 \pm 151.69$ & $-1.43 \pm 0.03$ & $12.03 \pm 0.90$ \\
\hline 042 & 02 & J032701.43-002207.1 & 2.319 & 0712-52199-0159 & $1727.30 \pm 179.78$ & $-1.60 \pm 0.03$ & $11.20 \pm 1.00$ \\
\hline 043 & 01 & J033029.75-005918.1 & 2.747 & 0414-51869-0004 & $1860.40 \pm 228.82$ & $-1.23 \pm 0.04$ & $9.48 \pm 1.55$ \\
\hline 043 & 02 & J033029.75-005918.1 & 2.747 & 0414-51901-0021 & $1654.68 \pm 195.10$ & $-0.98 \pm 0.04$ & $20.70 \pm 1.62$ \\
\hline 044 & 01 & J074123.74+190453.8 & 2.288 & 2074-53437-0325 & $866.49 \pm 71.04$ & $-1.08 \pm 0$ & $7.66 \pm 0.86$ \\
\hline 044 & 02 & J0741 & & 2915 & 852 & $-1.08 \pm 0$ & \\
\hline 045 & 01 & J074221.38+165740.3 & 2.538 & 2074-53437 & $1383.77 \pm 85.90$ & $-1.31 \pm 0.02$ & \\
\hline 045 & 02 & J074221.38+165740.3 & 2.538 & 2915-54497-0216 & $1553.70 \pm 96.80$ & $-1.25 \pm 0.02$ & $5.10 \pm 0.68$ \\
\hline 046 & 01 & J074818.62+272304.7 & 2.344 & 2075-53737-0043 & $658.23 \pm 76.70$ & $-1.29 \pm 0.04$ & $7.46 \pm 1.24$ \\
\hline 046 & 02 & J074818.62+272304.7 & 2.344 & $0-0055$ & $679.43 \pm 58.28$ & $-1.31 \pm 0.03$ & $7.36 \pm 0.94$ \\
\hline 047 & 01 & J075007.63+275708.0 & 2.364 & 2618-0071 & $2710.02 \pm 128.52$ & $-1.36 \pm 0.02$ & $23.93 \pm 0.62$ \\
\hline 047 & 02 & J075007.63+275708.0 & 2.364 & 2075-53737-0550 & $1799.98 \pm 68.30$ & $-1.03 \pm 0.02$ & $29.12 \pm 0.50$ \\
\hline 048 & 01 & J080049.70+092830.7 & 2.182 & 2419-54139-0262 & $1248.89 \pm 130.64$ & $-1.26 \pm 0.04$ & $9.03 \pm 1.15$ \\
\hline 048 & 02 & J080049.70+092830.7 & 2.182 & $2945-54505-0638$ & $1316.89 \pm 72.55$ & $-1.17 \pm 0.02$ & $11.08 \pm 0.60$ \\
\hline 049 & 01 & J080455.90+231501.8 & 2.162 & $1265-52705-0187$ & $1467.36 \pm 153.08$ & $-1.13 \pm 0.04$ & $16.28 \pm 1.08$ \\
\hline 049 & 02 & J080455.90+231501.8 & 2.162 & $1584-52943-0337$ & $1544.43 \pm 107.60$ & $-1.53 \pm 0.03$ & $15.41 \pm 0.75$ \\
\hline 050 & 01 & J081213.95+431715.9 & 1.742 & 0546-52205-0403 & $2010.89 \pm 109.20$ & $-1.68 \pm 0.06$ & $73.49 \pm 0.70$ \\
\hline 050 & 02 & & & & & $-1.68 \pm 0$ & $75.13 \pm 0.61$ \\
\hline 051 & 01 & J081822.63+434633.8 & 2.042 & 0547-51959-0122 & $2322.17 \pm 177.45$ & $-1.28 \pm 0.03$ & $2.78 \pm 0.87$ \\
\hline 051 & 02 & J081822.63+434633.8 & 2.042 & 0547-52207-0157 & $1937.76 \pm 107.42$ & $-1.26 \pm 0.02$ & $8.13 \pm 0.61$ \\
\hline 052 & 01 & J082238.64+420925.7 & 1.968 & 0761-52266-0244 & $670.99 \pm 81.22$ & $-0.69 \pm 0.06$ & $7.56 \pm 1.44$ \\
\hline 052 & 02 & J082238.64+420925.7 & 1.968 & 0761-54524-0279 & $1100.38 \pm 147.55$ & $-1.81 \pm 0.09$ & $3.73 \pm 0.99$ \\
\hline 053 & 01 & J083925.61+045420.2 & 2.447 & $1187-52708-0137$ & $949.11 \pm 126.67$ & $1.14 \pm 0.10$ & $42.31 \pm 1.67$ \\
\hline 053 & 02 & J083925.61+045420.2 & 2.447 & $1188-52650-0390$ & $1058.86 \pm 121.28$ & $1.48 \pm 0.10$ & $41.67 \pm 1.38$ \\
\hline 054 & 01 & J084255.92+223431.9 & 2.714 & 2084-53360-0502 & $2138.52 \pm 229.21$ & $-1.02 \pm 0.03$ & $19.41 \pm 1.17$ \\
\hline 054 & 02 & J084255.92+223431.9 & 2.714 & $3373-54940-0062$ & $2407.33 \pm 134.97$ & $-1.25 \pm 0.02$ & $12.08 \pm 0.59$ \\
\hline 055 & 01 & J090241.07+571829.0 & 2.070 & $0483-51942-0521$ & $708.01 \pm 123.95$ & $0.00 \pm 0.06$ & $15.65 \pm 1.76$ \\
\hline 055 & 02 & J090241.07+571829.0 & 2.070 & 0483-51902-0554 & $692.91 \pm 82.68$ & $-0.05 \pm 0.05$ & $18.90 \pm 1.26$ \\
\hline 056 & 01 & J091512.53+305014.9 & & & & $-1.12 \pm 0$ & $4.57 \pm 0.96$ \\
\hline 056 & 02 & J091512.53+305014.9 & 1.983 & $2401-53768-0472$ & $1287.03 \pm 62.89$ & $-1.13 \pm 0.02$ & $4.05 \pm 0.52$ \\
\hline 057 & 01 & J092015.68+350040.5 & 1.916 & $1273-52993-0630$ & $1187.70 \pm 107.89$ & $-1.28 \pm 0.06$ & $27.79 \pm 0.95$ \\
\hline 057 & 02 & J092015.68+350040.5 & 1.916 & $1274-52995-0284$ & $1090.35 \pm 99.96$ & $-1.34 \pm 0.06$ & $28.18 \pm 0.96$ \\
\hline 058 & 01 & J092527.71+151416.9 & 1.968 & $2440-53818-0622$ & $1039.65 \pm 96.15$ & $-1.39 \pm 0.05$ & $14.58 \pm 1.06$ \\
\hline 058 & 02 & J092527.71+151416.9 & 1.968 & 3192-54829-0504 & $1268.01 \pm 61.49$ & $-1.47 \pm 0.02$ & $8.45 \pm 0.57$ \\
\hline 059 & 01 & J092720.29+101627.0 & 1.929 & $1740-53050-0065$ & $983.36 \pm 103.77$ & $-2.25 \pm 0.08$ & $35.81 \pm 1.37$ \\
\hline 059 & 02 & J092720.29+101627.0 & 1.929 & $3319-54915-0425$ & $796.30 \pm 43.66$ & $-1.10 \pm 0.04$ & $34.60 \pm 0.74$ \\
\hline 060 & 01 & J093300.21+544905.2 & 1.825 & 0556-51991-0313 & $925.17 \pm 103.13$ & $-1.09 \pm 0.09$ & $10.55 \pm 1.34$ \\
\hline 060 & 02 & J093300.21+544905.2 & 1.825 & 0555-52266-0154 & $919.61 \pm 84.46$ & $-1.62 \pm 0.08$ & $7.42 \pm 1.05$ \\
\hline 061 & 01 & J093333.29+410522.8 & 2.153 & 0940-52670-0287 & $2118.21 \pm 226.18$ & $-1.12 \pm 0.04$ & $7.87 \pm 1.08$ \\
\hline 061 & 02 & J093333.29+410522.8 & 2.153 & 0939-52636-0034 & $2275.52 \pm 138.07$ & $-1.06 \pm 0.02$ & $6.47 \pm 0.65$ \\
\hline
\end{tabular}


Table 2. - continue

\begin{tabular}{|c|c|c|c|c|c|c|c|}
\hline $\begin{array}{l}\text { No. } \\
\text { (1) }\end{array}$ & $\begin{array}{l}\text { epoch } \\
\text { (2) }\end{array}$ & $\begin{array}{l}\text { name } \\
(3)\end{array}$ & $\begin{array}{c}\mathrm{z} \\
(4)\end{array}$ & $\begin{array}{c}\text { Plate-MJD-Fiber } \\
\text { (5) }\end{array}$ & $\begin{array}{l}L_{1500} \\
\text { (6) }\end{array}$ & $\begin{array}{l}\alpha \\
(7)\end{array}$ & $\begin{array}{l}\text { BAL EW } \\
(8)\end{array}$ \\
\hline 062 & 01 & J093548.50+363121.9 & 2.977 & $1275-52996-0145$ & $2526.87 \pm 224.46$ & $-1.42 \pm 0.03$ & $20.97 \pm 1.04$ \\
\hline 062 & 02 & J093548.50+363121.9 & 2.977 & $3223-54865-0303$ & $2310.33 \pm 159.98$ & $-1.04 \pm 0.02$ & $33.81 \pm 0.76$ \\
\hline 063 & 01 & J093620.52+004649.2 & 1.719 & 0476-52027-0442 & $1099.22 \pm 173.80$ & $-1.34 \pm 0.10$ & $4.75 \pm 1.67$ \\
\hline 063 & 02 & J093620.52+004649.2 & 1.719 & 0476-52314-0444 & $772.49 \pm 110.71$ & $-0.91 \pm 0.09$ & $6.41 \pm 1.51$ \\
\hline 064 & 01 & J093859.27+150118.6 & 2.193 & $2581-54085-0228$ & $1379.40 \pm 142.56$ & $-0.83 \pm 0.04$ & $4.16 \pm 1.12$ \\
\hline 064 & 02 & J093859.27+150118.6 & 2.193 & $3196-54834-0640$ & $1802.93 \pm 83.73$ & $-0.74 \pm 0.02$ & $1.28 \pm 0.48$ \\
\hline 065 & 01 & J094338.21-010019.3 & 2.377 & 0266-51630-0124 & $1639.51 \pm 149.56$ & $-0.77 \pm 0.04$ & $30.02 \pm 0.97$ \\
\hline 065 & 02 & J094338.21-010019.3 & 2.377 & 0266-51602-0131 & $1723.56 \pm 150.00$ & $-0.92 \pm 0.03$ & $31.13 \pm 0.93$ \\
\hline 066 & 01 & J094425.46+610934.4 & 2.271 & 0486-51910-0120 & $1121.96 \pm 112.69$ & $-0.55 \pm 0.04$ & $35.61 \pm 1.08$ \\
\hline 066 & 02 & J094425.46+610934.4 & 2.271 & 2403-53795-0124 & $1171.95 \pm 60.84$ & $-1.23 \pm 0.02$ & $32.36 \pm 0.52$ \\
\hline 067 & 01 & J094456.75+544117.9 & 1.895 & 0769-54530-0327 & $785.66 \pm 85.51$ & $-0.60 \pm 0.07$ & $6.59 \pm 1.14$ \\
\hline 067 & 02 & J094456.75+544117.9 & 1.895 & 3169-54821-0377 & $698.54 \pm 78.91$ & $-0.29 \pm 0.06$ & $5.57 \pm 1.15$ \\
\hline 068 & 01 & J094602.23+380059.3 & 2.068 & $1276-53035-0160$ & $1675.13 \pm 130.50$ & $-1.23 \pm 0.03$ & $21.74 \pm 0.83$ \\
\hline 068 & 02 & J094602.23+380059.3 & 2.068 & $3223-54865-0566$ & $1599.50 \pm 76.83$ & $-0.85 \pm 0.02$ & $23.75 \pm 0.52$ \\
\hline 069 & 01 & J095357.00+040039.2 & 2.427 & $0571-52286-0560$ & $1310.91 \pm 134.76$ & $-0.93 \pm 0.03$ & $19.92 \pm 1.12$ \\
\hline 069 & 02 & J095357.00+040039.2 & 2.427 & 0572-52289-0312 & $1306.36 \pm 127.44$ & $-1.09 \pm 0.03$ & $20.38 \pm 1.06$ \\
\hline 070 & 01 & J095901.24+550408.2 & 2.180 & $0945-52652-0$ & $1194.36 \pm 79$ & $-0.89 \pm 0$ & $19.17 \pm 0.87$ \\
\hline 070 & 02 & J095901.24+550408.2 & 2.180 & 31 & $1675.41 \pm 7$ & $-1.39 \pm 0$ & $12.73 \pm 0$ \\
\hline 071 & 01 & $\mathrm{~J} 100109.51+1334$ & 1.841 & 2584 & $1428.41 \pm 11$ & $-0.71 \pm 0$ & $4.96 \pm$ \\
\hline 071 & 02 & J100109.51+133433.6 & 1.841 & $3248-54880-0120$ & $1554.03 \pm 87.20$ & $-0.97 \pm 0.04$ & $5.13 \pm 0.58$ \\
\hline 072 & 01 & $\mathrm{~J} 100318.99+521506.3$ & 3.325 & 0903-52385-0177 & $3897.33 \pm 280.68$ & $-1.70 \pm 0.04$ & $23.17 \pm 0.87$ \\
\hline 072 & 02 & $\mathrm{~J} 100$ & 3.325 & & $3813.42 \pm 235.67$ & $-1.62 \pm 0.03$ & $22.77 \pm 0.94$ \\
\hline 073 & 01 & $\mathrm{~J} 100619.31+6$ & 2.001 & 3-0077 & $899.42 \pm 91.37$ & $-1.05 \pm 0.04$ & $36.20 \pm 1.08$ \\
\hline 073 & 02 & J100619.31+625334.9 & 2.001 & 3294-54918-0102 & $715.36 \pm 67.37$ & $-1.53 \pm 0.05$ & $30.09 \pm 1.06$ \\
\hline 074 & 01 & J100716.69+030438.6 & 2.124 & 0501-52235-0606 & $2689.48 \pm 125.81$ & $-1.23 \pm 0.02$ & $6.36 \pm 0.48$ \\
\hline 074 & 02 & $\mathrm{~J} 100716.69+030438.6$ & 2.124 & $3257-54888-0474$ & $1927.77 \pm 83.14$ & $-0.91 \pm 0.02$ & $10.64 \pm 0.44$ \\
\hline 075 & 01 & $\mathrm{~J} 100912.49+252055.2$ & 2.205 & 2406-54084-0170 & $793.51 \pm 60.54$ & $-0.85 \pm 0.03$ & $34.54 \pm 0.85$ \\
\hline 075 & 02 & $\mathrm{~J} 100912.49+252055.2$ & 2.205 & $2347-53757-0$ & $861.28 \pm 119$ & $-0.71 \pm 0$ & $31.59 \pm 1.54$ \\
\hline 076 & 01 & $5.11+032003.7$ & 2.145 & 05 & 37 & $-1.42 \pm$ & \\
\hline 076 & 02 & & & & & $-1.42 \pm$ & \\
\hline 077 & 01 & $2.04+430455.6$ & 2.420 & 514 & $5537.78 \pm 24$ & $-1.55 \pm 0.01$ & $8.24 \pm 0.46$ \\
\hline 077 & 02 & $\mathrm{~J} 101542.04+430455.6$ & 2.420 & $3287-54941-0433$ & $4284.78 \pm 154.82$ & $-1.02 \pm 0.01$ & $12.99 \pm 0.36$ \\
\hline 078 & 01 & $6.34+383817.3$ & 1.953 & $1427-52996$ & $853.61 \pm 65.06$ & $-0.89 \pm 0.04$ & $49.94 \pm 0.88$ \\
\hline 078 & 02 & J101616.34+383817.3 & 1.953 & $3262-54884-0356$ & $1008.76 \pm 62.58$ & $-0.59 \pm 0.04$ & $54.69 \pm 0.70$ \\
\hline 079 & 01 & J102106.78+303137.5 & 3.061 & 2351-53772-0409 & $2114.50 \pm 168.99$ & $-1.97 \pm 0.03$ & $7.78 \pm 1.01$ \\
\hline 079 & 02 & $\mathrm{~J} 102106.78+303137.5$ & 3.061 & 2351-53786-0409 & $2376.23 \pm 254.61$ & $-2.01 \pm 0.04$ & $10.03 \pm 1.23$ \\
\hline 080 & 01 & $\mathrm{~J} 102156.84+282735.8$ & 1.880 & 2351-53772-0128 & $610.67 \pm 77.81$ & $-0.80 \pm 0.09$ & $26.23 \pm 1.33$ \\
\hline 080 & 02 & $\mathrm{~J} 102156.84+282735.8$ & 1.880 & $3260-54883-0624$ & $542.65 \pm 55.27$ & $-0.93 \pm 0.08$ & $28.45 \pm 1.04$ \\
\hline 081 & 01 & $\mathrm{~J} 102250.16+483631.1$ & 2.069 & $0873-52347-0522$ & $1121.23 \pm 91.69$ & $-1.01 \pm 0.04$ & $31.33 \pm 1.03$ \\
\hline 081 & 02 & & & & & $-1.43 \pm 0.03$ & \\
\hline 082 & 01 & $\mathrm{~J} 102754.03+182221.6$ & 3.078 & $2591-54140-0$ & $2578.13 \pm 231.53$ & $-1.20 \pm 0.03$ & $27.76 \pm 1.00$ \\
\hline 082 & 02 & $\mathrm{~J} 102754.03+182221.6$ & 3.078 & 2868-54451-0518 & $2370.06 \pm 84.24$ & $-1.07 \pm 0$ & $31.29 \pm 0.46$ \\
\hline 083 & 01 & J103006.62+271325.9 & 1.734 & 2353-53794-0164 & $871.74 \pm 99.58$ & $-0.60 \pm 0.08$ & $41.39 \pm 1.29$ \\
\hline 083 & 02 & $\mathrm{~J} 103006.62+271325.9$ & 1.734 & $3261-54881-0582$ & $893.54 \pm 43.96$ & $-0.94 \pm 0.05$ & $43.90 \pm 0.60$ \\
\hline 084 & 01 & $\mathrm{~J} 104010.46+432811.6$ & 2.595 & 2567-54179-0020 & $2204.80 \pm 105.51$ & $-1.21 \pm 0.02$ & $20.61 \pm 0.59$ \\
\hline 084 & 02 & $\mathrm{~J} 104010.46+432811.6$ & 2.595 & $3258-54884-0219$ & $1708.79 \pm 115.30$ & $-0.98 \pm 0.02$ & $20.60 \pm 0.83$ \\
\hline 085 & 01 & $\mathrm{~J} 104945.36+285823.3$ & 2.154 & 2359-53800-0332 & $732.44 \pm 101.18$ & $-0.15 \pm 0.05$ & $42.83 \pm 1.42$ \\
\hline 085 & 02 & $\mathrm{~J} 104945.36+285823.3$ & 2.154 & 2359-53826-0332 & $764.55 \pm 112.34$ & $-0.18 \pm 0.05$ & $42.27 \pm 1.48$ \\
\hline 086 & 01 & $\mathrm{~J} 105012.58+001158.8$ & 2.206 & 2409-54210-0538 & $366.41 \pm 38.80$ & $-0.38 \pm 0.04$ & $5.27 \pm 1.19$ \\
\hline 086 & 02 & $\mathrm{~J} 105012.58+001158.8$ & 2.206 & 2569-54234-0554 & $445.01 \pm 48.59$ & $-0.71 \pm 0.04$ & $6.90 \pm 1.21$ \\
\hline 087 & 01 & $\mathrm{~J} 105416.51+512326.0$ & & & $1799.34 \pm 142.16$ & $-1.56 \pm 0.03$ & $10.81 \pm 0.86$ \\
\hline 087 & 02 & & & & & & \\
\hline 088 & 01 & J105657.54+492957.9 & 2.162 & 0876-52669-0014 & $2073.65 \pm 172.16$ & $-1.03 \pm 0.03$ & $1.91 \pm 0.88$ \\
\hline 088 & 02 & J105657.54+492957.9 & 2.162 & 0876-52346-0017 & $1211.93 \pm 138.04$ & $-1.67 \pm 0.04$ & $0.97 \pm 1.20$ \\
\hline 089 & 01 & $\mathrm{~J} 110015.55+271451.7$ & 3.339 & 2359-53800-0003 & $3164.53 \pm 191.43$ & $-1.22 \pm 0.04$ & $22.14 \pm 0.79$ \\
\hline 089 & 02 & $\mathrm{~J} 110015.55+271451.7$ & 3.339 & $2359-53826-0003$ & $3247.59 \pm 170.00$ & $-1.19 \pm 0.04$ & $21.17 \pm 0.82$ \\
\hline 090 & 01 & J110152.91+275838.6 & 2.865 & $2211-53786-0256$ & $2034.26 \pm 323.16$ & $-0.26 \pm 0.04$ & $8.94 \pm 1.42$ \\
\hline 090 & 02 & J110152.91+275838.6 & 2.865 & 2870-54534-0165 & $2008.90 \pm 132.62$ & $-0.11 \pm 0.02$ & $6.97 \pm 0.72$ \\
\hline 091 & 01 & $\mathrm{~J} 110208.59+660156.5$ & 2.064 & 0490-51929-0142 & $897.03 \pm 75.33$ & $-1.14 \pm 0.04$ & $24.04 \pm 0.89$ \\
\hline 091 & 02 & $\mathrm{~J} 110208.59+660156.5$ & 2.064 & $3171-54862-0164$ & $1034.75 \pm 68.61$ & $-1.04 \pm 0.03$ & $18.57 \pm 0.69$ \\
\hline 092 & 01 & $\mathrm{~J} 110427.08+054848.3$ & 3.006 & 0581-52353-0328 & $2714.96 \pm 233.04$ & $-2.29 \pm 0.03$ & $4.70 \pm 1.12$ \\
\hline 092 & 02 & $\mathrm{~J} 110427.08+054848.3$ & 3.006 & 0581-52356-0328 & $2849.43 \pm 230.02$ & $-2.23 \pm 0.03$ & $4.87 \pm 1.03$ \\
\hline
\end{tabular}


Table 2. - continue

\begin{tabular}{|c|c|c|c|c|c|c|c|}
\hline $\begin{array}{l}\text { No. } \\
\text { (1) }\end{array}$ & $\begin{array}{l}\text { epoch } \\
\text { (2) }\end{array}$ & $\begin{array}{l}\text { name } \\
(3)\end{array}$ & $\begin{array}{c}\mathrm{Z} \\
(4)\end{array}$ & $\begin{array}{c}\text { Plate-MJD-Fiber } \\
\text { (5) }\end{array}$ & $\begin{array}{l}L_{1500} \\
\text { (6) }\end{array}$ & $\begin{array}{c}\alpha \\
\text { (7) }\end{array}$ & $\begin{array}{l}\text { BAL EW } \\
\text { (8) }\end{array}$ \\
\hline 093 & 01 & $\mathrm{~J} 111313.29+102212.4$ & 2.247 & $1222-52763-0322$ & $4914.87 \pm 252.26$ & $-0.99 \pm 0.02$ & $8.25 \pm 0.53$ \\
\hline 093 & 02 & $\mathrm{~J} 111313.29+102212.4$ & 2.247 & 2393-54156-0420 & $6065.31 \pm 123.81$ & $-1.08 \pm 0.01$ & $7.90 \pm 0.22$ \\
\hline 094 & 01 & $\mathrm{~J} 111651.98+463508.6$ & 1.888 & $3216-54908-0385$ & $540.64 \pm 62.58$ & $-1.52 \pm 0.08$ & $14.73 \pm 1.18$ \\
\hline 094 & 02 & $\mathrm{~J} 111651.98+463508.6$ & 1.888 & 3216-54853-0399 & $581.58 \pm 51.68$ & $-1.41 \pm 0.06$ & $11.95 \pm 0.98$ \\
\hline 095 & 01 & $\mathrm{~J} 112239.20+602012.3$ & 2.227 & 0951-52398-0523 & $1142.37 \pm 128.40$ & $-1.50 \pm 0.04$ & $9.10 \pm 1.17$ \\
\hline 095 & 02 & $\mathrm{~J} 112239.20+602012.3$ & 2.227 & 3328-54964-0081 & $966.08 \pm 94.23$ & $-1.73 \pm 0.03$ & $5.71 \pm 0.93$ \\
\hline 096 & 01 & $\mathrm{~J} 112258.77+164540.3$ & 3.031 & 2499-54176-0308 & $8025.55 \pm 284.56$ & $-1.04 \pm 0.03$ & $42.12 \pm 0.45$ \\
\hline 096 & 02 & $\mathrm{~J} 112258.77+164540.3$ & 3.031 & $3327-54951-0153$ & $7874.83 \pm 162.16$ & $-0.81 \pm 0.03$ & $39.93 \pm 0.30$ \\
\hline 097 & 01 & $\mathrm{~J} 112703.06+450516.4$ & 1.857 & $1366-53063-0321$ & $1130.13 \pm 113.64$ & $-1.85 \pm 0.10$ & $20.09 \pm 1.06$ \\
\hline 097 & 02 & $\mathrm{~J} 112703.06+450516.4$ & 1.857 & $3215-54861-0399$ & $671.43 \pm 50.17$ & $-1.64 \pm 0.07$ & $12.46 \pm 0.78$ \\
\hline 098 & 01 & J112939.84+600728.9 & 1.724 & 0952-52409-0412 & $1091.60 \pm 108.85$ & $-1.44 \pm 0.07$ & $5.73 \pm 1.11$ \\
\hline 098 & 02 & J112939.84+600728.9 & 1.724 & $3211-54852-0424$ & $1024.48 \pm 71.53$ & $-1.71 \pm 0.05$ & $2.00 \pm 0.77$ \\
\hline 099 & 01 & $29.56+584803.8$ & 1.781 & 0952-52409-0150 & $778.03 \pm 98.26$ & $-1.23 \pm 0.09$ & $21.69 \pm 1.36$ \\
\hline 099 & 02 & $\mathrm{~J} 113529.56+584803.8$ & 1.781 & 2881-54502-0296 & $672.88 \pm 82.21$ & $-1.60 \pm 0.09$ & $30.81 \pm 1.37$ \\
\hline 100 & 01 & $\mathrm{~J} 113621.05+005021.2$ & 3.428 & 0282-51630-0535 & $8531.06 \pm 370.79$ & $-1.77 \pm 0.02$ & $6.83 \pm 0.46$ \\
\hline 100 & 02 & $\mathrm{~J} 113621.05+005021.2$ & 3.428 & 0282-51658-0535 & $8891.49 \pm 282.79$ & $-1.71 \pm 0.02$ & $6.31 \pm 0.38$ \\
\hline 101 & 01 & $\mathrm{~J} 115944.82+011206.9$ & 2.002 & 0285-51663-0530 & $5261.82 \pm 266.65$ & $-2.11 \pm 0.02$ & $18.10 \pm 0.53$ \\
\hline 101 & 02 & $\mathrm{~J} 115944.82+011206.9$ & 2.002 & 0285-51930-0540 & $4932.98 \pm 191.60$ & $-1.67 \pm 0.02$ & $18.12 \pm 0.40$ \\
\hline 102 & 01 & $\mathrm{~J} 120217.29+332108.7$ & 2.252 & 2089-53498-0292 & $1772.50 \pm 118.41$ & $-1.64 \pm 0.02$ & $8.47 \pm 0.71$ \\
\hline 102 & 02 & $\mathrm{~J} 120217.29+33210$ & 2.252 & $2095-53474-0$ & $1800.74 \pm$ & $-1.57 \pm$ & $9.41=$ \\
\hline 103 & 01 & $\mathrm{~J} 120653.39+492919.3$ & 1.845 & 0969-52442-0107 & $2508.88 \pm 130.53$ & $-1.71 \pm 0.05$ & $64.15 \pm 0.55$ \\
\hline 103 & 02 & J120653.39+492919.3 & 1.845 & 2919 & $2198.63 \pm 58.20$ & $-1.55 \pm 0.03$ & $59.46 \pm 0.28$ \\
\hline 104 & 01 & $\mathrm{~J} 120822.25+302423.9$ & 2.252 & 2230-53799-0353 & $2827.30 \pm 21$ & $-1.43 \pm 0.02$ & $5.31 \pm 0.73$ \\
\hline 104 & 02 & $\mathrm{~J} 120822.25+302423.9$ & 2.252 & $3181-54860-0025$ & $2552.20 \pm 11$ & $-1.21 \pm 0.01$ & $1.68 \pm 0.49$ \\
\hline 105 & 01 & $\mathrm{~J} 121$ & 2.413 & 41 & $2679.76 \pm$ & $-1.69 \pm$ & $23.20 \pm 0.82$ \\
\hline 105 & 02 & $\mathrm{~J} 121147.38+203402.4$ & 2.413 & 2918-54554-0622 & $2761.27 \pm 93.74$ & $-1.76 \pm 0.01$ & $17.27 \pm 0.39$ \\
\hline 106 & 01 & $\mathrm{~J} 121328.78-025617.8$ & 2.153 & 0332-52367-0065 & $2299.24 \pm 184.48$ & $-1.11 \pm 0.03$ & $13.76 \pm 0.85$ \\
\hline 106 & 02 & $\mathrm{~J} 121328.78-025617.8$ & 2.153 & 0333-52313-0315 & $2302.60 \pm 149.92$ & $-1.19 \pm 0.03$ & $11.93 \pm 0.73$ \\
\hline 107 & 01 & $\mathrm{~J} 122604.28+034317.8$ & 1.767 & 0519-52283-0565 & $1956.38 \pm 109.69$ & $-1.37 \pm 0.05$ & $20.78 \pm 0.58$ \\
\hline 107 & 02 & $\mathrm{~J} 122604.28+034317.8$ & 1.767 & 3253-54941-0202 & $2113.96 \pm 101.23$ & $-1.31 \pm 0.03$ & $21.63 \pm 0.50$ \\
\hline 108 & 01 & $\mathrm{~J} 122951.77+351929.9$ & 1.821 & 2010-53495-0309 & $1731.18 \pm 116$ & $-1.42 \pm 0$ & $18.34 \pm 0.79$ \\
\hline 108 & 02 & $\mathrm{~J} 122951.77+35192$ & 1.821 & & & $-1.66 \pm 0$ & $14.79 \pm 0.55$ \\
\hline 109 & 01 & $\mathrm{~J} 123303.50+620915.9$ & 1.839 & & $1419.77 \pm 1$ & $-1.45 \pm 0$ & $18.91 \pm 0.89$ \\
\hline 109 & 02 & $\mathrm{~J} 123303.50+620915.9$ & 1.839 & 0781-52373-0278 & $1502.92 \pm 152.26$ & $-1.52 \pm 0.08$ & $19.42 \pm 1.08$ \\
\hline 110 & 01 & $\mathrm{~J} 123411.74+615832.5$ & 1.949 & 0780-52370-0065 & $1047.71 \pm 127.36$ & $-1.04 \pm 0.07$ & $40.59 \pm 1.32$ \\
\hline 110 & 02 & $\mathrm{~J} 123411.74+615832.5$ & 1.949 & 0781-52373-0275 & $958.88 \pm 113.42$ & $-0.73 \pm 0.07$ & $38.02 \pm 1.29$ \\
\hline 111 & 01 & $\mathrm{~J} 123736.42+143640.1$ & 2.704 & $1768-53442-0038$ & $2075.61 \pm 219.11$ & $-1.00 \pm 0.03$ & $31.35 \pm 1.25$ \\
\hline 111 & 02 & $\mathrm{~J} 123736.42+143640.1$ & 2.704 & 3254-54889-0548 & $2426.53 \pm 129.76$ & $-1.23 \pm 0.02$ & $31.44 \pm 0.63$ \\
\hline 112 & 01 & $\mathrm{~J} 124140.08+131746.1$ & 2.143 & $1694-53472-0336$ & $1303.73 \pm 120.77$ & $-1.37 \pm 0.04$ & $10.71 \pm 1.07$ \\
\hline 112 & 02 & $\mathrm{~J} 124140.08+131746.1$ & 2.143 & $3255-54885-0205$ & $1096.03 \pm 72.40$ & $-1.21 \pm 0.02$ & $13.47 \pm 0.65$ \\
\hline 113 & 01 & $\mathrm{~J} 124551.44+010505.0$ & 2.809 & 0291-51660-0607 & $4162.68 \pm 267.66$ & $-1.54 \pm 0.02$ & $29.94 \pm 0.92$ \\
\hline 113 & 02 & $\mathrm{~J} 124551.44+010505.0$ & 2.809 & 0291-51928-0612 & $3921.53 \pm 236.91$ & $-1.61 \pm 0.02$ & $29.11 \pm 0.78$ \\
\hline 114 & 01 & $\mathrm{~J} 125950.76+183236.1$ & 2.248 & 2616-54499-0124 & $1083.87 \pm 122.29$ & $-1.04 \pm 0.04$ & $3.88 \pm 1.22$ \\
\hline 114 & 02 & $\mathrm{~J} 125950.76+183236.1$ & & & $1031.82 \pm 71.36$ & $-0.92 \pm 0$ & 0.76 \\
\hline 115 & 01 & $\mathrm{~J} 130136.12+000157.9$ & 1.783 & 0293-51994-0074 & $3457.46 \pm 161.29$ & $-1.54 \pm 0.05$ & $35.63 \pm 0.56$ \\
\hline 115 & 02 & $\mathrm{~J} 130136.12+000157.9$ & 1.783 & 0293-51689-0079 & $3261.62 \pm 129.30$ & $-1.72 \pm 0.04$ & $35.73 \pm 0.47$ \\
\hline 116 & 01 & $\mathrm{~J} 130221.80-004638.2$ & 2.704 & 0293-51689-0012 & $2020.28 \pm 259.70$ & $-0.83 \pm 0.04$ & $19.90 \pm 1.76$ \\
\hline 116 & 02 & $\mathrm{~J} 130221.80-004638.2$ & 2.704 & 0293-51994-0015 & $1901.44 \pm 217.58$ & $-0.92 \pm 0.04$ & $14.52 \pm 1.74$ \\
\hline 117 & 01 & J131416.43-015020.9 & 2.180 & 0340-51691-0508 & $1280.93 \pm 155.59$ & $-1.65 \pm 0.04$ & $7.17 \pm 1.30$ \\
\hline 117 & 02 & J131416.43-015020.9 & 2.180 & 0340-51990-0514 & $1386.06 \pm 116.92$ & $-1.86 \pm 0.03$ & $5.04 \pm 0.93$ \\
\hline 118 & 01 & $\mathrm{~J} 131433.83+032321.9$ & 2.255 & 0525-52029-0572 & $2105.90 \pm 201.20$ & $-1.75 \pm 0.03$ & $5.48 \pm 1.01$ \\
\hline 118 & 02 & $\mathrm{~J} 131433.83+032321.9$ & 2.255 & 0525-52295-0576 & $1720.59 \pm 137.72$ & $-1.46 \pm 0.02$ & $11.15 \pm 0.81$ \\
\hline 119 & 01 & $\mathrm{~J} 131505.89+590157.5$ & 1.932 & 0958-52410-0157 & $2256.73 \pm 137.19$ & $-0.90 \pm 0.04$ & $25.40 \pm 0.71$ \\
\hline 119 & 02 & $\mathrm{~J} 131505.89+590157.5$ & 1.932 & 3237-54883-0067 & $1889.68 \pm 78.10$ & $-0.63 \pm 0.02$ & $20.72 \pm 0.46$ \\
\hline 120 & 01 & $\mathrm{~J} 131714.21+010013.0$ & & & $3857.85 \pm 267.72$ & $-1.61 \pm 0.03$ & $23.92 \pm 0.88$ \\
\hline 120 & 02 & $\mathrm{~J} 131714.21+010013.0$ & & & & $-1.55 \pm 0.02$ & $26.54 \pm 0.76$ \\
\hline 121 & 01 & $\mathrm{~J} 131853.45+002211.5$ & 2.074 & 0296-51578-0383 & $1530.04 \pm 143.96$ & $-1.60 \pm 0.04$ & $19.34 \pm 1.00$ \\
\hline 121 & 02 & $\mathrm{~J} 131853.45+002211.5$ & 2.074 & 0296-51984-0390 & $1372.49 \pm 130.93$ & $-1.47 \pm 0.03$ & $21.71 \pm 0.99$ \\
\hline 122 & 01 & $\mathrm{~J} 131905.95+660415.7$ & 1.710 & 0496-51973-0260 & $1544.80 \pm 169.04$ & $-2.24 \pm 0.08$ & $9.63 \pm 1.14$ \\
\hline 122 & 02 & $\mathrm{~J} 131905.95+660415.7$ & 1.710 & 0496-51988-0260 & $1415.09 \pm 161.92$ & $-1.78 \pm 0.07$ & $7.41 \pm 1.15$ \\
\hline 123 & 01 & J132304.58-003856.5 & 1.827 & 0296-51578-0074 & $1363.05 \pm 108.92$ & $-1.36 \pm 0.07$ & $25.33 \pm 1.04$ \\
\hline 123 & 02 & J132304.58-003856.5 & 1.827 & 0296-51984-0076 & $1328.15 \pm 113.27$ & $-1.11 \pm 0.07$ & $22.89 \pm 1.08$ \\
\hline
\end{tabular}


$B A L$ variable regions

Table 2. - continue

\begin{tabular}{|c|c|c|c|c|c|c|c|}
\hline $\begin{array}{l}\text { No. } \\
\text { (1) }\end{array}$ & $\begin{array}{l}\text { epoch } \\
\text { (2) }\end{array}$ & $\begin{array}{l}\text { name } \\
(3)\end{array}$ & $\begin{array}{c}\mathrm{Z} \\
(4)\end{array}$ & $\begin{array}{l}\text { Plate-MJD-Fiber } \\
\text { (5) }\end{array}$ & $\begin{array}{l}L_{1500} \\
\text { (6) }\end{array}$ & $\begin{array}{c}\alpha \\
(7)\end{array}$ & $\begin{array}{l}\text { BAL EW } \\
\text { (8) }\end{array}$ \\
\hline 124 & 01 & $\mathrm{~J} 132422.54+245222.4$ & 2.363 & 2664-54524-0537 & $3194.67 \pm 168.12$ & $-1.38 \pm 0.02$ & $28.43 \pm 0.56$ \\
\hline 124 & 02 & $\mathrm{~J} 132422.54+245222.4$ & 2.363 & $3303-54950-0599$ & $2773.42 \pm 102.74$ & $-1.36 \pm 0.01$ & $27.57 \pm 0.37$ \\
\hline 125 & 01 & $\mathrm{~J} 132827.06+581836.8$ & 3.139 & 0960-52466-0304 & $2794.53 \pm 251.42$ & $-1.76 \pm 0.05$ & $11.45 \pm 1.38$ \\
\hline 125 & 02 & $\mathrm{~J} 132827.06+581836.8$ & 3.139 & 0960-52425-0318 & $2827.13 \pm 188.18$ & $-2.15 \pm 0.04$ & $14.99 \pm 0.87$ \\
\hline 126 & 01 & $\mathrm{~J} 133138.50+004221.1$ & 2.424 & 0298-51955-0374 & $2264.05 \pm 164.18$ & $-1.82 \pm 0.02$ & $11.14 \pm 0.78$ \\
\hline 126 & 02 & $\mathrm{~J} 133138.50+004221.1$ & 2.424 & 0298-51662-0376 & $2168.88 \pm 208.04$ & $-1.62 \pm 0.03$ & $6.24 \pm 1.10$ \\
\hline 127 & 01 & $\mathrm{~J} 133514.39+531805.8$ & 1.874 & $1041-52724-0039$ & $1578.48 \pm 127.97$ & $-1.17 \pm 0.06$ & $13.08 \pm 0.91$ \\
\hline 127 & 02 & $\mathrm{~J} 133514.39+531805.8$ & 1.874 & 3318-54951-0115 & $1395.43 \pm 83.67$ & $-0.99 \pm 0.04$ & $14.02 \pm 0.63$ \\
\hline 128 & 01 & $\mathrm{~J} 134101.28+083755.7$ & 2.494 & $1804-53886-0318$ & $1512.42 \pm 144.88$ & $0.30 \pm 0.08$ & $53.75 \pm 1.08$ \\
\hline 128 & 02 & $\mathrm{~J} 134101.28+083755.7$ & 2.494 & $2928-54614-0128$ & $1427.07 \pm 68.11$ & $-0.06 \pm 0.05$ & $56.01 \pm 0.52$ \\
\hline 129 & 01 & $\mathrm{~J} 134544.55+002810.7$ & 2.453 & 0300-51943-0382 & $1918.11 \pm 151.67$ & $-1.34 \pm 0.03$ & $20.84 \pm 0.90$ \\
\hline 129 & 02 & $\mathrm{~J} 134544.55+002810.7$ & 2.453 & 0300-51666-0426 & $2153.34 \pm 160.97$ & $-1.65 \pm 0.03$ & $17.78 \pm 0.83$ \\
\hline 130 & 01 & J135448.04+501137.9 & 2.141 & $1670-54553-($ & $722.58 \pm 8$ & $-0.57 \pm 0.05$ & $11.40 \pm 1.31$ \\
\hline 130 & 02 & J135448.04+501137.9 & 2.141 & $1670-53438-0486$ & $771.58 \pm 81.16$ & $-0.76 \pm 0.04$ & $5.88 \pm 1.16$ \\
\hline 131 & 01 & J135559.03-002413.6 & 2.337 & 0301-51641-0266 & $3095.13 \pm 179.97$ & $-1.68 \pm 0.02$ & $14.60 \pm 0.65$ \\
\hline 131 & 02 & J135559.03-002413.6 & 2.337 & 0301-51942-0267 & $2769.95 \pm 162.58$ & $-1.72 \pm 0.02$ & $15.86 \pm 0.67$ \\
\hline 132 & 01 & $\mathrm{~J} 135721.77+005501.1$ & 1.997 & 0301-51942-0408 & $2278.58 \pm 128.98$ & $-1.27 \pm 0.02$ & $8.54 \pm 0.59$ \\
\hline 132 & 02 & $\mathrm{~J} 135721.77+005501.1$ & 1.997 & 0301-51641-0411 & $2084.64 \pm 133.82$ & $-1.31 \pm 0.03$ & $8.32 \pm 0.66$ \\
\hline 133 & 01 & J135941.58+000851.9 & 1.736 & $0301-51942-0$ & $862.52 \pm 97.91$ & $-1.72 \pm 0.08$ & $5.32 \pm 1.17$ \\
\hline 133 & 02 & $\mathrm{~J} 13$ & 1.736 & 03 & 800 & $-1.39 \pm$ & $7.58 \pm$ \\
\hline 134 & 01 & $\mathrm{~J} 142333.56+573909.5$ & 1.870 & $0789-52342-0229$ & $789.71 \pm 102$ & $-0.73 \pm 0.10$ & $25.77 \pm 1.49$ \\
\hline 134 & 02 & $3.56+573909.5$ & 1.870 & 265 & $735.62 \pm 11$ & $-0.08 \pm 0.11$ & $24.84 \pm 1.74$ \\
\hline 135 & 01 & $\mathrm{~J} 142910.55+634603.6$ & 1.886 & 143 & $686.52 \pm 7$ & $-1.47 \pm 0.08$ & $9.11 \pm 1.24$ \\
\hline 135 & 02 & J142910.55+634603.6 & 1.886 & 2947 & $522.58 \pm 6$ & $-1.34 \pm 0.09$ & $10.04 \pm 1.34$ \\
\hline 136 & 01 & $\mathrm{~J} 143$ & 1.891 & & $02 \pm$ & $-1.04 \pm 0.03$ & $15.91 \pm 0.54$ \\
\hline 136 & 02 & $17.07+632701.7$ & 1.891 & $533-0370$ & $1876.33 \pm 13$ & $-1.41 \pm 0.05$ & $11.06 \pm 0.75$ \\
\hline 137 & 01 & $\mathrm{~J} 143130.03+5$ & 1.797 & 0790-52441-0245 & $2142.59 \pm 14$ & $-1.74 \pm 0.06$ & $23.75 \pm 0.80$ \\
\hline 137 & 02 & $\mathrm{~J} 143130.03+570138.8$ & 1.797 & 0790-52346-0257 & $2224.85 \pm 146.92$ & $-1.96 \pm 0.06$ & $25.38 \pm 0.75$ \\
\hline 138 & 01 & $\mathrm{~J} 143307.40+003319.0$ & 2.744 & 0306-51637-0546 & $1573.84 \pm 179.18$ & $-1.87 \pm 0.04$ & $17.83 \pm 1.57$ \\
\hline 138 & 02 & $\mathrm{~J} 143307.40+003319.0$ & 2.744 & 0306-51690-0558 & $1552.12 \pm 176.33$ & $-1.77 \pm 0.04$ & $17.49 \pm 1.84$ \\
\hline 139 & 01 & $\mathrm{~J} 143612.69+443812.6$ & 1.848 & $1288-52731-0$ & $896.37 \pm 125.93$ & $-0.62 \pm 0$ & $18.20 \pm 1.50$ \\
\hline 139 & 02 & $\mathrm{~J} 143612.69+443812.6$ & 1.848 & & & $-0.51 \pm 0$ & $14.97 \pm 1.30$ \\
\hline 140 & 01 & $\mathrm{~J} 143641.24+00$ & 1.86 & & $.35 \pm \varepsilon$ & $-1.19 \pm 0$ & $28.91 \pm 0.97$ \\
\hline 140 & 02 & $\mathrm{~J} 143641.24+001558.9$ & & $0-0629$ & $1058.77 \pm 106.35$ & $-1.32 \pm 0.09$ & $31.47 \pm 1.17$ \\
\hline 141 & 01 & $\mathrm{~J} 143758.06+011119.5$ & 2.045 & 0307-51663-0443 & $1022.18 \pm 1$ & $-0.66 \pm 0.04$ & $24.68 \pm 1.18$ \\
\hline 141 & 02 & $\mathrm{~J} 1437$ & 2.045 & 0536 & $1014.26 \pm 11$ & $-0.80 \pm 0.04$ & $26.18 \pm 1.20$ \\
\hline 142 & 01 & $\mathrm{~J} 143907.51-010616.7$ & 1.819 & 1663-0089 & $539.73 \pm 116.89$ & $-0.27 \pm 0.14$ & $12.58 \pm 2.53$ \\
\hline 142 & 02 & $\mathrm{~J} 143907.51-010616.7$ & 1.819 & 0919-52409-0566 & $674.14 \pm 115.50$ & $-0.35 \pm 0.11$ & $10.88 \pm 1.90$ \\
\hline 143 & 01 & $\mathrm{~J} 144136.54+632519.4$ & 1.779 & 0609-52339-0322 & $1067.87 \pm 136.16$ & $-1.70 \pm 0.10$ & $13.63 \pm 1.38$ \\
\hline 143 & 02 & $\mathrm{~J} 144136.54+632519.4$ & 1.779 & 2947-54533-0153 & $730.82 \pm 76.55$ & $-2.36 \pm 0.08$ & $7.84 \pm 1.23$ \\
\hline 144 & 01 & $\mathrm{~J} 144351.38+560325.6$ & 2.275 & 0791-52347-0247 & $1532.55 \pm 145.80$ & $-1.60 \pm 0.03$ & $11.38 \pm 1.07$ \\
\hline 144 & 02 & $\mathrm{~J} 144351.38+560325.6$ & 2.275 & 0791-52435-0247 & $1632.08 \pm 169.46$ & $-1.62 \pm 0.04$ & $10.54 \pm 1.15$ \\
\hline 145 & 01 & J144412.36+582636.9 & 2.336 & 0790-52441-0583 & $1187.20 \pm 149.14$ & $-0.58 \pm 0.04$ & $2.63 \pm 1.32$ \\
\hline 145 & 02 & $\mathrm{~J} 144412.36+582636.9$ & & & & $-0.95 \pm 0$ & \\
\hline 146 & 01 & $\mathrm{~J} 144514.86-002358.1$ & 2.237 & & $3403.12 \pm 95.96$ & $0.21 \pm 0.02$ & $24.90 \pm 0.27$ \\
\hline 146 & 02 & $\mathrm{~J} 144514.86-002358.1$ & 2.237 & 2909-54653-0222 & $3109.81 \pm 142.56$ & $0.21 \pm 0.02$ & $24.98 \pm 0.48$ \\
\hline 147 & 01 & $\mathrm{~J} 144935.96+631836.0$ & 1.735 & 0609-52339-0538 & $891.96 \pm 130.81$ & $-1.12 \pm 0.10$ & $17.81 \pm 1.66$ \\
\hline 147 & 02 & $\mathrm{~J} 144935.96+631836.0$ & 1.735 & 2947-54533-0044 & $615.93 \pm 76.12$ & $-1.45 \pm 0.08$ & $16.42 \pm 1.51$ \\
\hline 148 & 01 & $\mathrm{~J} 145110.68+040925.2$ & 1.732 & 0588-52029-0440 & $1295.74 \pm 167.75$ & $-1.52 \pm 0.09$ & $19.43 \pm 1.37$ \\
\hline 148 & 02 & $\mathrm{~J} 145110.68+040925.2$ & 1.732 & 0588-52045-0440 & $1408.12 \pm 120.28$ & $-1.66 \pm 0.07$ & $19.36 \pm 0.93$ \\
\hline 149 & 01 & $\mathrm{~J} 145353.45+040124.0$ & 2.088 & 0588-52029-0488 & $1917.34 \pm 147.12$ & $-1.69 \pm 0.03$ & $10.69 \pm 0.99$ \\
\hline 149 & 02 & $\mathrm{~J} 145353.45+040124.0$ & 2.088 & 0588-52045-0496 & $2106.68 \pm 102.42$ & $-1.82 \pm 0.02$ & $10.19 \pm 0.70$ \\
\hline 150 & 01 & $\mathrm{~J} 145943.02+010601.5$ & 2.092 & 0310-51616-0393 & $2071.46 \pm 188.94$ & $-0.97 \pm 0.03$ & $2.97 \pm 0.97$ \\
\hline 150 & 02 & $\mathrm{~J} 145943.02+010601.5$ & 2.092 & 0538-52029-0012 & $2210.66 \pm 160.70$ & $-1.58 \pm 0.03$ & $3.39 \pm 0.79$ \\
\hline 151 & 01 & $\mathrm{~J} 150033.52+003353.6$ & & & $3455.47 \pm 259.64$ & $-1.11 \pm 0.02$ & $12.07 \pm 0.78$ \\
\hline 151 & 02 & $.52+003353.6$ & & & & $-0.53 \pm 0.03$ & 18.26 \\
\hline 152 & 01 & $\mathrm{~J} 150332.93+440120.6$ & 2.049 & $1676-53147-0028$ & $1118.21 \pm 104.63$ & $-0.68 \pm 0.04$ & $16.02 \pm 1.19$ \\
\hline 152 & 02 & $\mathrm{~J} 150332.93+440120.6$ & 2.049 & $1677-53148-0358$ & $1205.95 \pm 91.43$ & $-0.83 \pm 0.04$ & $18.93 \pm 1.02$ \\
\hline 153 & 01 & J150428.59-002015.9 & 1.865 & 0310-51616-0170 & $1359.99 \pm 154.39$ & $-1.30 \pm 0.09$ & $9.28 \pm 1.28$ \\
\hline 153 & 02 & J150428.59-002015.9 & 1.865 & 0310-51990-0199 & $1261.01 \pm 131.14$ & $-1.34 \pm 0.08$ & $9.30 \pm 1.12$ \\
\hline 154 & 01 & $\mathrm{~J} 150659.88+420652.7$ & 1.868 & $1291-52735-0489$ & $1156.12 \pm 101.45$ & $-1.54 \pm 0.08$ & $14.99 \pm 1.00$ \\
\hline 154 & 02 & $\mathrm{~J} 150659.88+420652.7$ & 1.868 & $1291-52738-0489$ & $1187.00 \pm 112.53$ & $-1.65 \pm 0.08$ & $16.55 \pm 1.03$ \\
\hline
\end{tabular}


Table 2. - continue

\begin{tabular}{|c|c|c|c|c|c|c|c|}
\hline $\begin{array}{l}\text { No. } \\
\text { (1) }\end{array}$ & $\begin{array}{l}\text { epoch } \\
\text { (2) }\end{array}$ & $\begin{array}{l}\text { name } \\
(3)\end{array}$ & $\begin{array}{c}\mathrm{Z} \\
(4)\end{array}$ & $\begin{array}{c}\text { Plate-MJD-Fiber } \\
\text { (5) }\end{array}$ & $\begin{array}{l}L_{1500} \\
\text { (6) }\end{array}$ & $\begin{array}{c}\alpha \\
\text { (7) }\end{array}$ & $\begin{array}{l}\text { BAL EW } \\
\text { (8) }\end{array}$ \\
\hline 155 & 01 & $\mathrm{~J} 152057.81+461641.3$ & 1.962 & $1050-52721-0629$ & $648.26 \pm 113.38$ & $0.72 \pm 0.07$ & $55.97 \pm 1.92$ \\
\hline 155 & 02 & $\mathrm{~J} 152057.81+461641.3$ & 1.962 & $1331-52766-0248$ & $630.92 \pm 91.87$ & $0.39 \pm 0.08$ & $60.70 \pm 1.73$ \\
\hline 156 & 01 & $\mathrm{~J} 152149.78+010236.4$ & 2.231 & 0313-51673-0339 & $1365.55 \pm 164.68$ & $-1.48 \pm 0.04$ & $6.09 \pm 1.25$ \\
\hline 156 & 02 & $\mathrm{~J} 152149.78+010236.4$ & 2.231 & 2953-54560-0209 & $2686.50 \pm 183.33$ & $-1.83 \pm 0.02$ & $3.84 \pm 0.70$ \\
\hline 157 & 01 & $\mathrm{~J} 153045.76+383952.3$ & 2.022 & $1293-52765-0068$ & $2835.80 \pm 153.79$ & $-1.74 \pm 0.02$ & $16.74 \pm 0.54$ \\
\hline 157 & 02 & $\mathrm{~J} 153045.76+383952.3$ & 2.022 & $1294-52753-0329$ & $2418.72 \pm 167.68$ & $-1.36 \pm 0.03$ & $14.27 \pm 0.75$ \\
\hline 158 & 01 & J153715.74+582933.9 & 2.590 & 0615-52345-0577 & $6004.06 \pm 393.01$ & $-0.68 \pm 0.02$ & $16.86 \pm 0.59$ \\
\hline 158 & 02 & $\mathrm{~J} 153715.74+582933.9$ & 2.590 & $0615-52347-0585$ & $6259.21 \pm 269.68$ & $-0.75 \pm 0.01$ & $16.87 \pm 0.42$ \\
\hline 159 & 01 & $\mathrm{~J} 154359.43+535903.1$ & 2.371 & 0616-52374-0097 & $6736.25 \pm 391.38$ & $-1.73 \pm 0.02$ & $4.99 \pm 0.68$ \\
\hline 159 & 02 & J154359.43+535903.1 & 2.371 & 0616-52442-0120 & $6930.08 \pm 274.79$ & $-1.56 \pm 0.01$ & $4.20 \pm 0.41$ \\
\hline 160 & 01 & $\mathrm{~J} 160649.23+451051.6$ & 2.826 & 0814-52370-0355 & $2815.78 \pm 261.00$ & $-0.58 \pm 0.03$ & $17.28 \pm 1.31$ \\
\hline 160 & 02 & $\mathrm{~J} 160649.23+451051.6$ & 2.826 & 0814-52443-0359 & $2792.49 \pm 275.76$ & $-0.60 \pm 0.03$ & $18.92 \pm 1.31$ \\
\hline 161 & 01 & J163023.69+390841.7 & 2.008 & $1172-52759-0008$ & $881.66 \pm 139.10$ & $-0.03 \pm 0.06$ & $14.28 \pm 1.58$ \\
\hline 161 & 02 & J163023.69+390841.7 & 2.008 & $1173-52790-0453$ & $790.73 \pm 134.68$ & $0.09 \pm 0.07$ & $12.60 \pm 1.91$ \\
\hline 162 & 01 & $\mathrm{~J} 164419.97+274447.0$ & 3.068 & $1690-53475-0350$ & $5126.54 \pm 224.13$ & $-1.39 \pm 0.02$ & $24.99 \pm 0.59$ \\
\hline 162 & 02 & $\mathrm{~J} 164419.97+274447.0$ & 3.068 & 2949-54557-0603 & $4163.99 \pm 231.63$ & $-1.70 \pm 0.02$ & $27.11 \pm 0.65$ \\
\hline 163 & 01 & J164424.98+274136.5 & 3.893 & $1690-53475-0342$ & $9125.81 \pm 840.91$ & $-1.07 \pm 0.03$ & $5.79 \pm 0.57$ \\
\hline 163 & 02 & J164424.98+274136.5 & 3.893 & 2949-54557-0602 & $7408.34 \pm 746.56$ & $-1.33 \pm 0.03$ & $4.58 \pm 0.60$ \\
\hline 164 & 01 & $\mathrm{~J} 165443.30+372008.0$ & 1.928 & 0632-52071-0125 & $1332.06 \pm 136.03$ & $-1.58 \pm 0.05$ & $3.47 \pm 1.06$ \\
\hline 164 & 02 & $\mathrm{~J} 165443.30+37200$ & 1.928 & 0820 & $1157.64 \pm 9$ & $-1.79 \pm 0$ & $3.72=$ \\
\hline 165 & 01 & $\mathrm{~J} 165631.20+353259.0$ & 2.039 & 0819-52409-0006 & $857.54 \pm 126.85$ & $-0.30 \pm 0.06$ & $32.15 \pm 1.58$ \\
\hline 165 & 02 & $\mathrm{~J} 165631.20+35$ & 2.039 & 438-0092 & $1036.77 \pm 8$ & $-0.80 \pm 0.04$ & $35.35 \pm 0.94$ \\
\hline 166 & 01 & $\mathrm{~J} 170105.08+372347.3$ & 1.959 & 0820-52438-0614 & $796.09 \pm 108.81$ & $-0.73 \pm 0.06$ & $18.23 \pm 1.29$ \\
\hline 166 & 02 & $\mathrm{~J} 170105.08+372347.3$ & 1.959 & $0820-52405-0638$ & $838.88 \pm 97.70$ & $-1.10 \pm 0.06$ & $16.51 \pm 1.26$ \\
\hline 167 & 01 & $\mathrm{~J} 170633.06+61$ & 2.012 & 035 & $1272.88 \pm$ & $-1.76 \pm$ & $9.93 \pm 1.12$ \\
\hline 167 & 02 & J170633.06+615715.1 & 2.012 & $695-0556$ & $1321.14 \pm 137.66$ & $-1.64 \pm 0.04$ & $11.44 \pm 0.98$ \\
\hline 168 & 01 & $\mathrm{~J} 170931.00+630357.2$ & 2.395 & 0352-51789-0310 & $3799.17 \pm 193.13$ & $-1.20 \pm 0.02$ & $26.52 \pm 0.58$ \\
\hline 168 & 02 & $\mathrm{~J} 170931.00+630357.2$ & 2.395 & 0352-51694-0311 & $3023.91 \pm 174.46$ & $-0.91 \pm 0.03$ & $29.68 \pm 0.67$ \\
\hline 169 & 01 & $\mathrm{~J} 171731.04+621912.0$ & 2.118 & 0352-51789-0209 & $1199.22 \pm 122.94$ & $-1.53 \pm 0.04$ & $10.12 \pm 1.11$ \\
\hline 169 & 02 & $\mathrm{~J} 171731.04+621912.0$ & 2.118 & 0352-51694-0215 & $1113.01 \pm 138.59$ & $-1.66 \pm 0.05$ & $9.99 \pm 1.33$ \\
\hline 170 & 01 & $\mathrm{~J} 172012.40+545601.1$ & 2.100 & 0357-51813-0189 & $1923.35 \pm 103$ & $-1.78 \pm 0$ & $14.19 \pm 0.59$ \\
\hline 170 & 02 & $\mathrm{~J} 172012.40+545601.1$ & 2.100 & & $2071.91 \pm 10$ & $-2.02 \pm 0$ & $14.55 \pm 0.49$ \\
\hline 171 & 01 & $\mathrm{~J} 173802.90+535047.2$ & 1.918 & & $989.13 \pm 11$ & $-0.35 \pm 0.06$ & $6.12 \pm 1.21$ \\
\hline 171 & 02 & $\mathrm{~J} 173802.90+535047.2$ & 1.918 & 0360-51780-0134 & $703.79 \pm 148.22$ & $0.70 \pm 0.10$ & $2.17 \pm 2.26$ \\
\hline 172 & 01 & J212127.78-081737.5 & 1.906 & 0640-52178-0286 & $1276.19 \pm 169.68$ & $-0.84 \pm 0.06$ & $2.42 \pm 1.31$ \\
\hline 172 & 02 & J212127.78-081737.5 & 1.906 & 0640-52200- & $1189.13 \pm 180.42$ & $-0.83 \pm 0.08$ & $1.95 \pm 1.45$ \\
\hline 173 & 01 & J213113.93-083913.5 & 1.983 & 0641-52176-0297 & $632.51 \pm 115.90$ & $-0.33 \pm 0.08$ & $22.50 \pm 1.99$ \\
\hline 173 & 02 & J213113.93-083913.5 & 1.983 & 0641-52199-0299 & $732.44 \pm 103.17$ & $-0.65 \pm 0.07$ & $22.87 \pm 1.63$ \\
\hline 174 & 01 & $\mathrm{~J} 213138.07-002537.8$ & 1.837 & 0989-52468-0273 & $591.30 \pm 115.95$ & $-0.25 \pm 0.13$ & $34.51 \pm 2.15$ \\
\hline 174 & 02 & $\mathrm{~J} 213138.07-002537.8$ & 1.837 & $1963-54331-0238$ & $301.29 \pm 44.97$ & $0.36 \pm 0.08$ & $17.94 \pm 1.60$ \\
\hline 175 & 01 & J213138.93-070013.4 & 2.048 & 0641-52176-0386 & $1272.71 \pm 116.96$ & $-1.55 \pm 0.04$ & $29.72 \pm 1.07$ \\
\hline 175 & 02 & J213138.93-070013.4 & 2.048 & 0641-52199-0390 & $1258.61 \pm 101.25$ & $-1.46 \pm 0.04$ & $32.74 \pm 0.97$ \\
\hline 176 & 01 & J213508.59-075502.1 & 1.799 & 0641-52176-0108 & $1611.66 \pm 128.10$ & $-1.45 \pm 0.06$ & $6.42 \pm 0.94$ \\
\hline 176 & 02 & $\mathrm{~J} 213508.59-075502.1$ & & & $1778.34 \pm 140.06$ & $-1.71 \pm 0$ & $8.97 \pm 0.93$ \\
\hline 177 & 01 & J213648.17-001546.6 & 2.180 & 0989-52468-0104 & $1169.71 \pm 128.27$ & $-1.46 \pm 0.04$ & $13.46 \pm 1.23$ \\
\hline 177 & 02 & J213648.17-001546.6 & 2.180 & $1152-52941-0317$ & $1320.84 \pm 103.66$ & $-1.42 \pm 0.03$ & $8.75 \pm 0.83$ \\
\hline 178 & 01 & $\mathrm{~J} 221555.98+010127.0$ & 2.241 & $1104-52912-0530$ & $1838.20 \pm 148.25$ & $-1.66 \pm 0.03$ & $13.56 \pm 0.87$ \\
\hline 178 & 02 & $\mathrm{~J} 221555.98+010127.0$ & 2.241 & 3146-54773-0609 & $1671.33 \pm 105.68$ & $-1.21 \pm 0.02$ & $8.65 \pm 0.66$ \\
\hline 179 & 01 & $\mathrm{~J} 222107.29+125627.2$ & 1.732 & 0736-52210-0027 & $1291.86 \pm 161.31$ & $-1.06 \pm 0.08$ & $24.85 \pm 1.38$ \\
\hline 179 & 02 & $\mathrm{~J} 222107.29+125627.2$ & 1.732 & 0736-52221-0031 & $1514.98 \pm 151.15$ & $-1.48 \pm 0.07$ & $23.80 \pm 1.06$ \\
\hline 180 & 01 & J224248.92-085822.8 & 2.390 & 0722-52224-0075 & $1594.81 \pm 161.98$ & $-1.80 \pm 0.03$ & $11.72 \pm 1.03$ \\
\hline 180 & 02 & J224248.92-085822.8 & 2.390 & $0723-52201-0358$ & $1654.45 \pm 160.01$ & $-1.78 \pm 0.03$ & $11.31 \pm 0.96$ \\
\hline 181 & 01 & J224324.59-005330.7 & 1.895 & 0378-52146-0216 & $1001.88 \pm 150.58$ & $-0.65 \pm 0.09$ & $19.33 \pm 1.58$ \\
\hline 181 & 02 & J224324.59-005330.7 & 1.895 & 0675-52590-0208 & $1159.24 \pm 110.38$ & $-0.78 \pm 0.05$ & $17.35 \pm 1.03$ \\
\hline 182 & 01 & J225706.17-002532.8 & & & $519.08 \pm 117.48$ & $1.05 \pm 0.13$ & $26.30 \pm 2.44$ \\
\hline 182 & 02 & $\mathrm{~J} 225706.17-002532.8$ & & & & $1.02 \pm 0.12$ & $20.28 \pm 2.71$ \\
\hline 183 & 01 & J231105.54-102837.5 & 3.194 & $0726-52226-0130$ & $6948.05 \pm 210.14$ & $-1.13 \pm 0.02$ & $43.91 \pm 0.38$ \\
\hline 183 & 02 & $\mathrm{~J} 231105.54-102837.5$ & 3.194 & 0726-52207-0133 & $7419.36 \pm 378.42$ & $-1.08 \pm 0.03$ & $43.52 \pm 0.58$ \\
\hline 184 & 01 & $\mathrm{~J} 231958.70-002449.3$ & 1.890 & 0382-51816-0064 & $1329.00 \pm 130.74$ & $-0.12 \pm 0.06$ & $21.62 \pm 1.05$ \\
\hline 184 & 02 & J231958.70-002449.3 & 1.890 & 0680-52200-0261 & $1562.23 \pm 130.52$ & $-0.72 \pm 0.06$ & $24.83 \pm 0.92$ \\
\hline 185 & 01 & J232840.99-085315.8 & 1.729 & 0646-52523-0446 & $762.37 \pm 100.71$ & $-1.47 \pm 0.10$ & $41.52 \pm 1.65$ \\
\hline 185 & 02 & J232840.99-085315.8 & 1.729 & $3145-54801-0367$ & $1301.68 \pm 46.78$ & $-1.57 \pm 0.03$ & $40.79 \pm 0.47$ \\
\hline
\end{tabular}


Table 2. - continue

\begin{tabular}{lccccccc}
\hline \hline $\begin{array}{l}\text { No. } \\
(1)\end{array}$ & $\begin{array}{c}\text { epoch } \\
(2)\end{array}$ & $\begin{array}{c}\text { name } \\
(3)\end{array}$ & $\begin{array}{c}\mathrm{z} \\
(4)\end{array}$ & $\begin{array}{c}\text { Plate-MJD-Fiber } \\
(5)\end{array}$ & $\begin{array}{c}L_{1500} \\
(6)\end{array}$ & $\begin{array}{c}\alpha \\
(7)\end{array}$ & $\begin{array}{c}\text { BAL EW } \\
(8)\end{array}$ \\
\hline 186 & 01 & $\mathrm{~J} 234711.44-103742.4$ & 1.800 & $0648-52559-0098$ & $2954.64 \pm 116.50$ & $-0.07 \pm 0.04$ & $49.98 \pm 0.55$ \\
186 & 02 & $\mathrm{~J} 234711.44-103742.4$ & 1.800 & $2311-54331-0216$ & $2922.86 \pm 71.76$ & $-0.36 \pm 0.03$ & $51.92 \pm 0.41$ \\
187 & 01 & $\mathrm{~J} 235702.54-004824.0$ & 3.013 & $0387-51791-0246$ & $2437.82 \pm 209.11$ & $-1.31 \pm 0.04$ & $18.02 \pm 1.10$ \\
187 & 02 & $\mathrm{~J} 235702.54-004824.0$ & 3.013 & $0685-52203-0317$ & $2321.58 \pm 213.74$ & $-1.38 \pm 0.04$ & $20.08 \pm 1.21$ \\
188 & 01 & $\mathrm{~J} 235859.47-002426.2$ & 1.759 & $0387-51791-0181$ & $942.02 \pm 96.73$ & $-0.98 \pm 0.08$ & $15.85 \pm 1.25$ \\
188 & 02 & $\mathrm{~J} 235859.47-002426.2$ & 1.759 & $0685-52203-0164$ & $760.98 \pm 105.55$ & $-0.43 \pm 0.09$ & $21.50 \pm 1.53$ \\
\hline
\end{tabular}

Table 3. The subsample of 43 two-epoch spectra with identified variable BAL regions. Col. (1) is the No. of the two-epoch spectra. Col. (2) is the name. Col. (3) is the redshift. Col. (4) is one observation of Plate-MJD-Fiber. Col. (5) is another observation of Plate-MJD-Fiber. Col. (6) is the bolometric luminosity in units of erg s${ }^{-1}$. Col. (7) is the $\mathrm{Mg}$ II -based black hole mass in units of $M_{\odot}$. Col. (8) is the Eddington ratio. Col. (9) is $\Delta E W$ of all variable BAL regions in two-epoch different spectra in units of $\AA$. Col. (10) is the boundary of all variable BAL regions $\left(V_{\max }, V_{\min }\right)$ in units of $\mathrm{km} \mathrm{s}^{-1}$.

\begin{tabular}{|c|c|c|c|c|c|c|c|c|c|}
\hline $\begin{array}{l}\mathrm{N} \\
(1)\end{array}$ & $\begin{array}{l}\text { name } \\
\text { (2) }\end{array}$ & $\begin{array}{c}\mathrm{z} \\
(3) \\
\end{array}$ & $\begin{array}{c}\text { Plate-MJD-Fiber }{ }^{1} \\
\text { (4) }\end{array}$ & $\begin{array}{l}\text { Plate-MJD-Fiber }{ }^{2} \\
\text { (5) }\end{array}$ & $\begin{array}{c}\log L_{b o l} \\
(6)\end{array}$ & $\begin{array}{c}\log M_{B H} \\
\text { (7) }\end{array}$ & $\begin{array}{c}L_{b o l} / L_{E d d} \\
(8)\end{array}$ & $\begin{array}{c}\Delta E W^{\text {vary }} \\
(9)\end{array}$ & $\begin{array}{c}\operatorname{Vary}\left(V_{\max }, V_{\text {min }}\right) \\
(10)\end{array}$ \\
\hline 001 & J002710.06-094435.3 & 2.070 & 0653-52145-0556 & $3105-54825-0310$ & 46.96 & $8.87 \pm 0.19$ & 0.98 & $-8.45 \pm 0.54$ & $(27311.7,22275.5) ;(5036.2,2324.4)$ \\
\hline 002 & J003312.25+155442.4 & 1.937 & 0417-51821-0576 & 3133-54789-0379 & 46.23 & $8.86 \pm 0.19$ & 0.19 & $-4.17 \pm 0.59$ & $(21113.3,19951.1) ;(14333.8,12203.1)$ \\
\hline 003 & J004527.68+143816.1 & 1.992 & 0419-51812-0105 & 0419-51868-0106 & 47.44 & $9.70 \pm 0.03$ & 0.43 & $+0.61 \pm 0.17$ & $(7360.6,6198.4)$ \\
\hline 004 & J005419.99+002727.9 & 2.490 & 0394-51876-0514 & 3111-54800-0509 & 47.07 & - & - & $-2.76 \pm 0.29$ & $(8329.1,6585.8) ;(4455.1,3099.2)$ \\
\hline 005 & J010859.53-105757.8 & 1.807 & 0659-52199-0099 & 3109-54833-0003 & 46.74 & $8.93 \pm 0.11$ & 0.51 & $+5.03 \pm 0.74$ & $(28861.3,27311.7) ;(21500.7,20338.5) ;(11234.6,10072.4)$ \\
\hline 006 & J012603.62-100114.8 & 2.302 & 0661-52163-0114 & 2878-54465-0274 & 46.93 & - & - & $+2.93 \pm 0.46$ & $(6585.8,3292.9)$ \\
\hline 007 & J020006.31-003709.7 & 2.141 & 0403-51871-0070 & 2866-54478-0197 & 46.65 & $10.14 \pm 0.14$ & 0.03 & $+6.68 \pm 0.48$ & $(20338.5,18014.1) ;(16851.9,13365.3) ;(12590.5,11234.6)$ \\
\hline 008 & J021818.14-092153.5 & 1.880 & 0668-52162-0218 & 3122-54821-0201 & 47.08 & $9.80 \pm 0.13$ & 0.15 & $+0.76 \pm 0.21$ & $(12009.4,10847.2)$ \\
\hline 009 & J022036.27-081242.9 & 2.004 & 0668-52162-0547 & 3122-54821-0482 & 46.94 & $9.24 \pm 0.12$ & 0.40 & $-2.07 \pm 0.35$ & $(27311.7,26149.5) ;(23825.1,21888.1)$ \\
\hline 010 & J022349.24+004727.8 & 2.335 & 0704-52205-0459 & $3127-54835-0338$ & 46.43 & - & - & $+0.89 \pm 0.35$ & $(11428.3,10459.8)$ \\
\hline 011 & J023252.80-001351.1 & 2.033 & 0705-52200-0063 & 3126-54804-0205 & 46.70 & $9.24 \pm 0.19$ & 0.23 & $-6.65 \pm 0.56$ & $(10266.1,6585.8) ;(6198.4,4261.4)$ \\
\hline 012 & $\mathrm{~J} 074221.38+165740.3$ & 2.538 & 2074-53437-0207 & 2915-54497-0216 & 46.94 & - & - & $-1.20 \pm 0.24$ & $(23631.4,22275.5)$ \\
\hline 013 & J075007.63+275708.0 & 2.364 & 1059-52618-0071 & 2075-53737-0550 & 46.94 & - & - & $+6.43 \pm 0.47$ & $(23825.1,18401.5) ;(10072.4,6973.2)$ \\
\hline 014 & J080455.90+231501.8 & 2.162 & $1265-52705-0187$ & 1584-52943-0337 & 46.79 & $9.28 \pm 0.23$ & 0.26 & $-1.92 \pm 0.45$ & $(24018.8,21888.1)$ \\
\hline 015 & J081213.95+431715.9 & 1.742 & 0546-52205-0403 & 0547-51959-0284 & 46.95 & $9.37 \pm 0.10$ & 0.30 & $+4.58 \pm 0.60$ & $(28086.5,24212.5)$ \\
\hline 016 & J081822.63+434633.8 & 2.042 & 0547-51959-0122 & 0547-52207-0157 & 46.97 & $9.49 \pm 0.06$ & 0.24 & $+2.95 \pm 0.38$ & $(13946.4,10847.2)$ \\
\hline 017 & J082238.64+420925.7 & 1.968 & 0761-52266-0244 & 0761-54524-0279 & 46.56 & $9.44 \pm 0.12$ & 0.10 & $-2.78 \pm 0.44$ & $(8910.2,7941.7)$ \\
\hline 018 & J084255.92+223431.9 & 2.714 & 2084-53360-0502 & 3373-54940-0062 & 46.91 & - & - & $-4.81 \pm 0.60$ & $(20338.5,16270.8) ;(15883.4,14140.1)$ \\
\hline 019 & J092527.71+151416.9 & 1.968 & $2440-53818-0622$ & 3192-54829-0504 & 46.53 & $9.05 \pm 0.21$ & 0.24 & $-4.62 \pm 0.52$ & $(18788.9,16077.1) ;(9297.6,7941.7)$ \\
\hline 020 & J092720.29+101627.0 & 1.929 & $1740-53050-0065$ & $3319-54915-0425$ & 46.74 & $9.43 \pm 0.06$ & 0.16 & $-1.19 \pm 0.35$ & $(6004.7,4842.5)$ \\
\hline 021 & J093548.50+363121.9 & 2.977 & $1275-52996-0145$ & 3223-54865-0303 & 47.10 & - & - & $+10.56 \pm 0.91$ & $(19951.1,17820.4) ;(16270.8,11234.6) ;(9491.3,7941.7)$ \\
\hline 022 & J094602.23+380059.3 & 2.068 & $1276-53035-0160$ & $3223-54865-0566$ & 46.84 & $9.38 \pm 0.19$ & 0.23 & $+2.95 \pm 0.38$ & $(18207.8,16270.8) ;(9297.6,8135.4)$ \\
\hline 023 & J095901.24+550408.2 & 2.180 & 0945-52652-0436 & $3169-54821-0627$ & 46.79 & $9.61 \pm 0.12$ & 0.12 & $-5.10 \pm 0.49$ & $(10459.8,4261.4)$ \\
\hline 024 & $\mathrm{~J} 100716.69+030438.6$ & 2.124 & 0501-52235-0606 & 3257-54888-0474 & 47.10 & $9.42 \pm 0.36$ & 0.38 & $+4.37 \pm 0.28$ & $(20725.9,19176.3) ;(18788.9,16270.8)$ \\
\hline 025 & J101542.04+430455.6 & 2.420 & $1218-52709-0514$ & $3287-54941-0433$ & 47.48 & - & - & $+5.35 \pm 0.24$ & $(8716.5,6198.4) ;(5423.6,3680.3)$ \\
\hline 026 & $\mathrm{~J} 101616.34+383817.3$ & 1.953 & $1427-52996-0479$ & $3262-54884-0356$ & 46.38 & $9.06 \pm 0.21$ & 0.17 & $+6.01 \pm 0.65$ & $(25374.7,23437.7) ;(21694.4,19563.7) ;(19176.3,17820.4)$ \\
\hline 027 & $\mathrm{~J} 102754.03+182221.6$ & 3.078 & 2591-54140-0453 & 2868-54451-0518 & 47.13 & - & - & $+1.10 \pm 0.28$ & $(13171.6,11815.7)$ \\
\hline 028 & $\mathrm{~J} 110208.59+660156.5$ & 2.064 & 0490-51929-0142 & 3171-54862-0164 & 46.59 & $9.10 \pm 0.25$ & 0.25 & $-4.90 \pm 0.52$ & $(16851.9,15689.7) ;(15302.3,12396.8)$ \\
\hline 029 & $\mathrm{~J} 112239.20+602012.3$ & 2.227 & 0951-52398-0523 & 3328-54964-0081 & 46.66 & $9.29 \pm 0.15$ & 0.19 & $-4.66 \pm 0.61$ & $(22275.5,18788.9)$ \\
\hline 030 & $\mathrm{~J} 112258.77+164540.3$ & 3.031 & 2499-54176-0308 & 3327-54951-0153 & 47.35 & - & - & $+1.31 \pm 0.20$ & $(19757.4,18401.5) ;(8135.4,7166.9)$ \\
\hline 031 & $\mathrm{~J} 112703.06+450516.4$ & 1.857 & 1366-53063-0321 & 3215-54861-0399 & 46.67 & $9.47 \pm 0.14$ & 0.13 & $-2.89 \pm 0.42$ & $(5617.3,4648.8) ;(1937.0,774.8)$ \\
\hline 032 & J120653.39+492919.3 & 1.845 & 0969-52442-0107 & 2919-54537-0170 & 47.08 & $9.65 \pm 0.05$ & 0.22 & $-1.70 \pm 0.25$ & $(22662.9,21500.7) ;(10847.2,9297.6)$ \\
\hline 033 & $\mathrm{~J} 120822.25+302423.9$ & 2.252 & 2230-53799-0353 & $3181-54860-0025$ & 47.18 & - & - & $-1.29 \pm 0.28$ & $(18014.1,17045.6) ;(14140.1,13171.6)$ \\
\hline 034 & J121147.38+203402.4 & 2.413 & 2610-54476-0341 & 2918-54554-0622 & 47.00 & - & - & $-6.04 \pm 0.52$ & $(22081.8,21113.3) ;(13365.3,10653.5) ;(10266.1,7360.6) ;(6198.4,5229.9)$ \\
\hline 035 & J122604.28+034317.8 & 1.767 & 0519-52283-0565 & 3253-54941-0202 & 47.12 & $9.63 \pm 0.07$ & 0.25 & $+1.01 \pm 0.20$ & $(12009.4,10653.5)$ \\
\hline 036 & J123736.42+143640.1 & 2.704 & $1768-53442-0038$ & 3254-54889-0548 & 46.83 & - & - & $+0.98 \pm 0.27$ & $(22275.5,21307.0)$ \\
\hline 037 & J130136.12+000157.9 & 1.783 & 0293-51994-0074 & 0293-51689-0079 & 47.16 & $9.83 \pm 0.08$ & 0.17 & $+0.41 \pm 0.12$ & $(13752.7,12396.8)$ \\
\hline 038 & $\mathrm{~J} 131433.83+032321.9$ & 2.255 & 0525-52029-0572 & 0525-52295-0576 & 46.93 & - & - & $+3.34 \pm 0.45$ & $(7360.6,6004.7) ;(4842.5,3874.0)$ \\
\hline 039 & $\mathrm{~J} 131505.89+590157.5$ & 1.932 & 0958-52410-0157 & $3237-54883-0067$ & 47.10 & $9.69 \pm 0.05$ & 0.20 & $-2.47 \pm 0.32$ & $(10459.8,7941.7) ;(4067.7,2905.5)$ \\
\hline 040 & $\mathrm{~J} 135721.77+005501.1$ & 1.997 & 0301-51942-0408 & 0301-51641-0411 & 47.03 & $9.39 \pm 0.11$ & 0.34 & $-2.94 \pm 0.36$ & $(26149.5,23050.3)$ \\
\hline 041 & $\mathrm{~J} 143117.07+632701.7$ & 1.891 & 0499-51988-0105 & 2947-54533-0370 & 47.17 & $9.62 \pm 0.06$ & 0.28 & $-1.29 \pm 0.29$ & $(18595.2,16851.9)$ \\
\hline 042 & $\mathrm{~J} 150033.52+003353.6$ & 2.438 & 0310-51616-0363 & 0310-51990-0388 & 46.68 & - & - & $+7.25 \pm 0.58$ & $(21500.7,20338.5) ;(19370.0,14140.1)$ \\
\hline 043 & J232840.99-085315.8 & 1.729 & 0646-52523-0446 & 3145-54801-0367 & 46.69 & $9.43 \pm 0.06$ & 0.14 & $-2.21 \pm 0.47$ & $(14721.2,13365.3)$ \\
\hline
\end{tabular}

